\title{
Blood flow modeling reveals improved collateral artery performance during the regenerative period in mammalian hearts
}

Suhaas Anbazhakan

Stanford University

Pamela Rios Coronado

Stanford University

Ana Natalia Sy-Quia

Stanford University

Lek Wei Seow

Stanford University

Aubrey Hands

Stanford University

Mingming Zhao

Stanford University

Melody Dong

Stanford University

Martin Pfaller

Stanford University

Brian Raftrey

Stanford University

Christopher Cook

Stanford University

Daniel Bernstein

Stanford University

Koen Nieman

Stanford University

Anca Pasca

Stanford University

Alison Marsden

Stanford University

Kristy Red-Horse ( $\nabla$ kredhors@stanford.edu )

Stanford University https://orcid.org/0000-0003-1541-601X 


\section{Article}

Keywords: Collateral arteries, blood flow, coronary artery disease, heart regeneration, computational fluid dynamics

Posted Date: November 18th, 2021

DOI: https://doi.org/10.21203/rs.3.rs-1083222/v1

License: (1) This work is licensed under a Creative Commons Attribution 4.0 International License.

Read Full License

Version of Record: A version of this preprint was published at Nature Cardiovascular Research on August 12th, 2022. See the published version at https://doi.org/10.1038/s44161-022-00114-9. 
Blood flow modeling reveals improved collateral artery performance during the regenerative period in mammalian hearts

4 Suhaas Anbazhakan ${ }^{1,7}$, Pamela E. Rios Coronado 2,7 , Ana Natalia L. Sy-Quia ${ }^{2}$, Anson 5 Seow $^{2}$, Aubrey M. Hands ${ }^{2}$, Mingming Zhao ${ }^{3,4}$, Melody L. Dong ${ }^{1}$, Martin Pfaller ${ }^{1,3}$, Brian 6 C. Raftrey ${ }^{2}$, Christopher K. Cook ${ }^{2}$, Daniel Bernstein ${ }^{3,4}$, Koen Nieman ${ }^{5}$, Anca M. Pașca ${ }^{3}$, 7 Alison L. Marsden ${ }^{1,3,8,{ }^{*}, \text { Kristy Red-Horse }}{ }^{2,6,8,{ }^{*}}$

${ }^{1}$ Department of Bioengineering, Stanford University, Stanford, CA 94305, USA.

10 'Department of Biology, Stanford University, Stanford, CA 94305, USA.

11 'Department of Pediatrics, Stanford University School of Medicine, Stanford, CA, 94305

$12{ }^{4}$ Stanford Cardiovascular Institute, Stanford University School of Medicine, Stanford, CA 13 94305, USA

14 Departments of Cardiovascular Medicine and Radiology, School of Medicine, Stanford 15 University, Stanford, CA, 94305, USA

$16{ }^{6}$ Institute for Stem Cell Biology and Regenerative Medicine, Stanford University School

17 of Medicine, Stanford, CA 94305, USA.

$18{ }^{7}$ These authors contributed equally.

$19{ }^{8}$ These authors contributed equally.

$21{ }^{*}$ Corresponding author

22 Email: kredhors@stanford.edu (KR-H) and amarsden@stanford.edu (ALM) 
Collateral arteries are a vessel subtype that bridges two artery branches, forming a natural bypass that can deliver blood flow downstream of an occlusion. These bridges in the human heart are associated with better outcomes during coronary artery disease. We recently found that their rapid development in neonates supports heart regeneration, while the non-regenerative adult heart displays slow and minimal collateralization. Thus, inducing robust collateral artery networks could serve as viable treatment for cardiac ischemia, but reaching this goal requires more knowledge on their developmental mechanisms and functional capabilities. Here, we use whole-organ imaging and 3D computational fluid dynamics (CFD) modeling to identify the spatial architecture of and predict blood flow through collaterals in neonate and adult hearts. We found that neonate collaterals are more numerous, larger in diameter, and, even when similar in size/number, are predicted to more effectively re-perfuse an occluded coronary network when compared to adults. CFD analysis revealed that collaterals perform better in neonates because of decreased differential pressures along their coronary artery tree. Furthermore, testing of various collateral configurations indicated that larger, more proximal collaterals are more beneficial than many smaller ones, identifying a target architecture for therapeutic interventions. Morphometric analysis revealed how the coronary artery network expands during postnatal growth. Vessel

42 diameters do not scale with cardiac muscle growth. Instead, the coronary tree expands

43 solely by adding additional branches of a set length, a burst of which occurs during

44 murine puberty. Finally, we compared mouse structural and functional data to human

45 hearts. Surprisingly, fetal human hearts possessed a very large number of small, but 46 mature, smooth muscle cell covered collaterals while angiogram data indicated adult 47 patients with chronic coronary occlusions contained at least two. Comparing size ratios 48 with modeled mouse data suggested low re-perfusion capabilities of the embryonic 49 collaterals but higher functional benefits of those in diseased adults. Our unique 50 interdisciplinary approach allowed us to quantify the functional significance of collateral 51 arteries during heart regeneration and repair-a critical step towards realizing their 52 therapeutic potential. 
Cardiovascular disease, including coronary artery disease (CAD), is the leading cause of death worldwide ${ }^{1}$. Atherosclerosis causes coronary arteries to become partially or completely occluded, decreasing blood flow to the myocardium and jeopardizing cardiac muscle function and viability. Current treatments include percutaneous interventions and coronary artery bypass graft surgery, but these are highly invasive and a significant number are unsuccessful, especially in diffuse multi-vessel CAD, which calls for new treatments ${ }^{2}$. Humans and some other mammals can develop specialized blood vessels called collateral arteries that function as natural coronary bypasses.

63 Collateral arteries are defined as an artery segment directly bridging two artery

64 branches without intervening capillaries, such that they directly provide blood flow distal

65 to a coronary blockage. Although only a minority of adult humans have functionally significant collateral arteries, clinical observations indicate that they can successfully shunt blood around a stenosis to protect against myocardial ischemia and reduce the risk of cardiac death ${ }^{3-6}$. Thus, inducing collateral development could be a promising therapeutic approach for treating $C A D^{7}$. However, a major roadblock to this goal is the severe lack of knowledge about collateral developmental mechanisms and their ability to restore blood flow.

While studies have characterized the presence or absence of native collateral arteries across different mammals ${ }^{8}$, mice are the most common model for investigating

74 their function during cardiac injury, usually through surgically-induced myocardial

75 infarctions (MI) $)^{9-13}$. Mice do not generally have pre-existing collateral arteries, but they

76 can be observed in adults by 7 days post-MI when using vascular filling approaches, i.e.

77 Microfil injection into the vasculature. This method detects 6-10 collaterals per adult

78 heart at approximately $18 \mu \mathrm{m}$ in diameter ${ }^{10}$. Genetic deletions in chemokine receptors

79 that inhibit macrophages reduces collateral numbers ${ }^{10}$. Furthermore, mouse strains with

80 decreased collateral development have genetic variants that lower Rabep2 expression,

81 which encodes a protein involved in VEGFR2 endosomal trafficking and signaling ${ }^{14}$.

82 Thus, mice have been a useful model for understanding various aspects of collateral

83 biology. 
We recently used a different technique to identify collaterals-whole-mount 85 immunofluorescence - coupled with lineage tracing and mouse genetics to identify the 86 cellular and molecular mechanisms driving collateral development post-MI ${ }^{9}$. We found

87 that, in the regenerating neonate heart, collaterals form post-MI when arterial endothelial cells migrate into the infarct zone in response to hypoxia-induced CXCL12 and coalesce into collateral arteries. This process was termed artery reassembly and did not occur in the non-regenerative adult heart, suggesting that the collaterals observed during vascular filling (described above) utilized a different mechanism. Exogenous CXCL12 application induced artery reassembly in adults to create collaterals up to $40 \mu \mathrm{m}$ in diameter. Although these collaterals were positively correlated with heart regeneration and repair, and vascular filling methods established direct connections, a detailed description of how blood flows through these relatively small vascular connections is required to fully understand the functional capabilities and therapeutic potential of collateral arteries.

How structural parameters affect collateral hemodynamics in these injury models remains an unanswered question due to technical barriers of directly imaging blood flow. Clinical measurements of collateral flow rely on qualitative assessments from angiograms or indirect pressure measurements ${ }^{15-17}$. More accurate measurements of collateral flow in humans are not only invasive, but somewhat unreliable since conclusive relationships cannot be made without knowing the number and size of all collaterals, many of which cannot currently be imaged in the human heart via angiogram. Visualizing blood flow is even more difficult in experimental animals due to their small size. Conclusions regarding collateral flow are usually reached from ex-vivo 107 data, but not without significant limitations. Methods include: 1. Filling coronary vessels 108 through the aorta (Microfil casting, $\mu \mathrm{CT}$ and fluorescent conjugates) ${ }^{18-23}$, which creates 109 a non-cell specific volumetric map of coronary vessels, and 2 . Whole mount 110 immunostaining fixed hearts from postnatal transgenic mice ${ }^{9}$. None of these 111 approaches provide a precise picture of how flowing blood will distribute through 112 collaterals.

113 Because the physical laws governing fluid motion are known, computational fluid 114 dynamics (CFD) modeling tools can directly and precisely estimate blood flow. CFD has 
115 contributed to patient-specific surgical and treatment planning in numerous human 116 cardiovascular diseases ${ }^{24-28}$. CFD modeling has also been applied to the cardiovascular 117 systems of various animals to estimate hemodynamic forces that would otherwise be 118 difficult to directly measure ${ }^{29-32}$. In mice, computational studies have modeled blood 119 flow in the retinal vasculature, thoracic aorta, and even feto-placental vessels for the 120 purpose of defining how hemodynamic forces influence arterial remodeling events at the 121 cellular and molecular levels ${ }^{33-35}$. An additional major advantage of CFD modeling is the 122 ability to systematically alter certain parameters while keeping others constant, leading 123 to rapid conclusions on the reparative capabilities of different vascular architectures ${ }^{36}$. 124 To obtain correct estimates from CFD modeling, it is critical to have high125 resolution images of a vascular network with intact volumetric dimensions. To date, the 126 vascular labeling methods utilized have not had the resolution required to generate 127 detailed anatomic models suitable for CFD modeling. However, recent innovations in 128 tissue clearing and whole-organ microscopy now provide the possibility of generating sufficiently high resolution images suitable for CFD model building ${ }^{37-40}$. Thus, CFD is 130 perfectly poised to push forward our understanding of collateral function.

In this study, we sought to interrogate how different collateral configurations 132 affect blood flow post-injury. We optimized whole-organ immunostaining and clearing to 133 label and image the entire intact artery tree, allowing quantification of hemodynamic 134 forces via CFD using high-fidelity 3D models constructed in the neonate and adult. We 135 computationally generated virtual occlusions and various collateral configurations, 136 keeping other model parameters fixed, to measure levels of flow restoration. The results 137 showed that naturally forming collaterals in adult mouse hearts perform poorly and 138 restore little flow. The virtual equivalent of CXCL12-induced collaterals performs better 139 but remain sub-optimal. In contrast, naturally forming collaterals in neonate hearts are 140 highly restorative because the structural parameters of the early coronary tree and 141 cardiac output at this stage results in lower overall pressure loss along the coronary 142 tree. We additionally investigated collateral arteries in human hearts by generating 143 whole-organ images of fetal hearts and analyzing angiograms from patients with chronic 144 coronary occlusions. Surprisingly, we found both fetal hearts contained greater than 40 145 mature, smooth muscle covered collateral arteries, while only an average of two 
146 collaterals with measurable flow in patient angiograms. Comparing diameters to CFD

147 mouse models where flow restoration was quantified suggested that human fetal

148 collaterals may be too small to be functionally significant and that those in patients may

149 lie between the capabilities of neonate and adult mouse collaterals. In total, the

150 combination of whole-organ artery labeling with 3D CFD modeling provides a powerful

151 tool to accurately analyze hemodynamic forces in collateral arteries to broaden our

152 understanding of their functional significance and therapeutic potential.

Results

\section{Imaging the entire coronary artery tree in neonate and adult mouse hearts}

To utilize 3D CFD for modeling coronary blood flow at high resolution and with

157 controlled parameter perturbations, we required a method to image the entire intact

158 artery tree in three dimensions. A whole-organ immunostaining and clearing method

159 based on iDISCO was optimized for cardiac tissue using postnatal day 6 (P6) mice ${ }^{37,39}$,

160 which allowed us to image smooth muscle actin ( $\alpha$-SMA)-positive arterial smooth

161 muscle cells throughout the heart using Light sheet microscopy (Fig. 1a). The signal-to-

162 noise ratio of $\alpha-S M A$ staining was high, allowing sharp contrast of arteries throughout

163 the entire myocardium (Fig. 1b). Rendering the arterial immunolabeling in 3D using

164 Imaris software revealed vast improvements over our previous method ${ }^{9}$. Specifically,

165 the 3D architecture was retained (Fig. 1C), and we could fully observe the septal artery

$166(\mathrm{SpA})$ in addition to the left (LCA) and right (RCA) coronary arteries (Fig 1 $\left.\mathrm{C}_{\mathrm{ii}}\right)$. Another

167 improvement was the ability to fully immunolabel and image intact adult hearts (Fig. 1d).

168 Immunostaining of $\alpha$-SMA labeled arterial vessels throughout the entire adult heart (Fig.

169 1e), even deep within the septum (Fig. 1f). Co-staining with arterial endothelial marker,

170 Connexin40, confirmed extensive overlap in both neonate and adults (Fig. $1 \mathrm{~b}_{\text {iii-v }}$ and

171 data not shown). Qualitatively, the density of arteries is much greater in neonates

172 compared to adult (Compare Fig.1a and 1d), and more branches were detected when

173 compared to $\mu \mathrm{CT}$ methods, both in neonatal and adult stages ${ }^{41}$. These data

174 demonstrate that iDISCO and Light sheet microscopy together, are capable of

175 effectively labeling and imaging smooth muscle covered arteries throughout neonatal 
Neonate (P6) Wild Type



Adult (P60) Wild Type
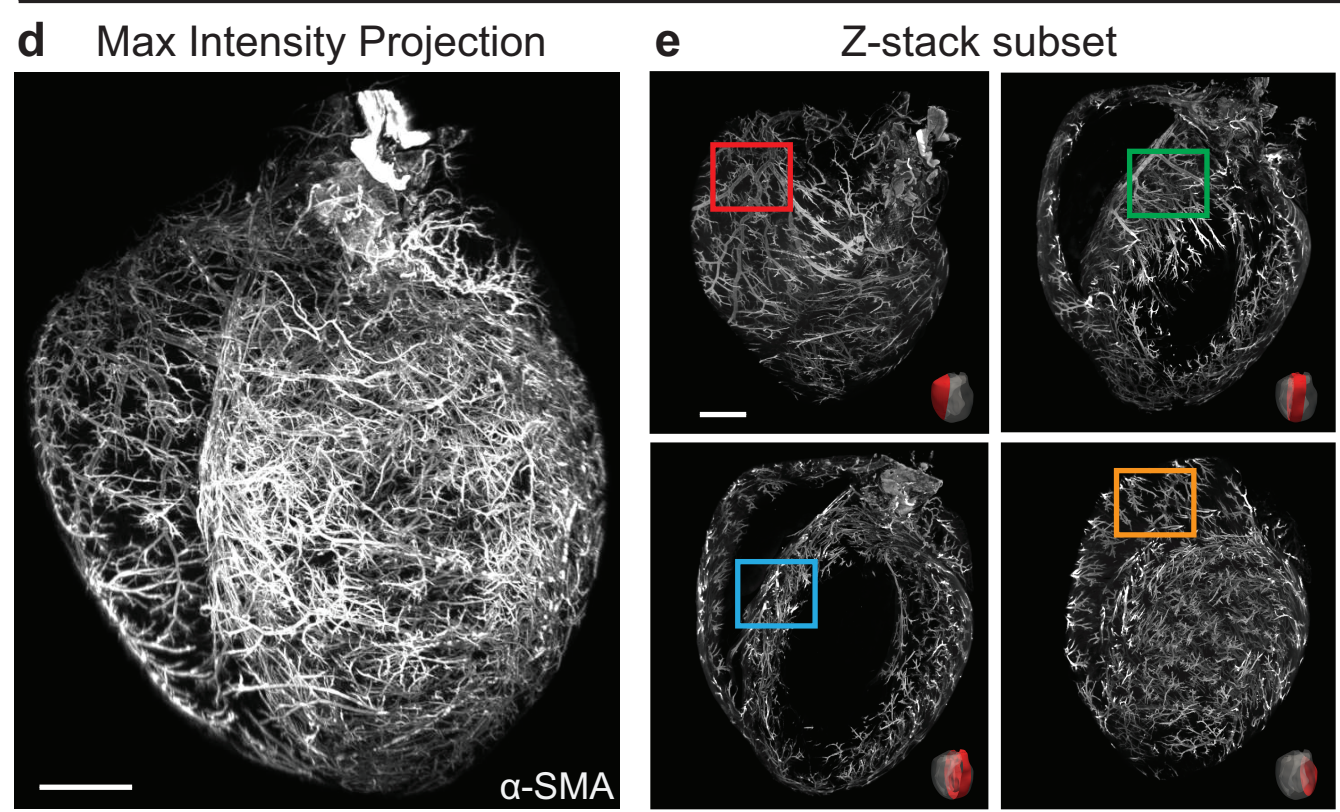

f $\mathrm{ROI}$
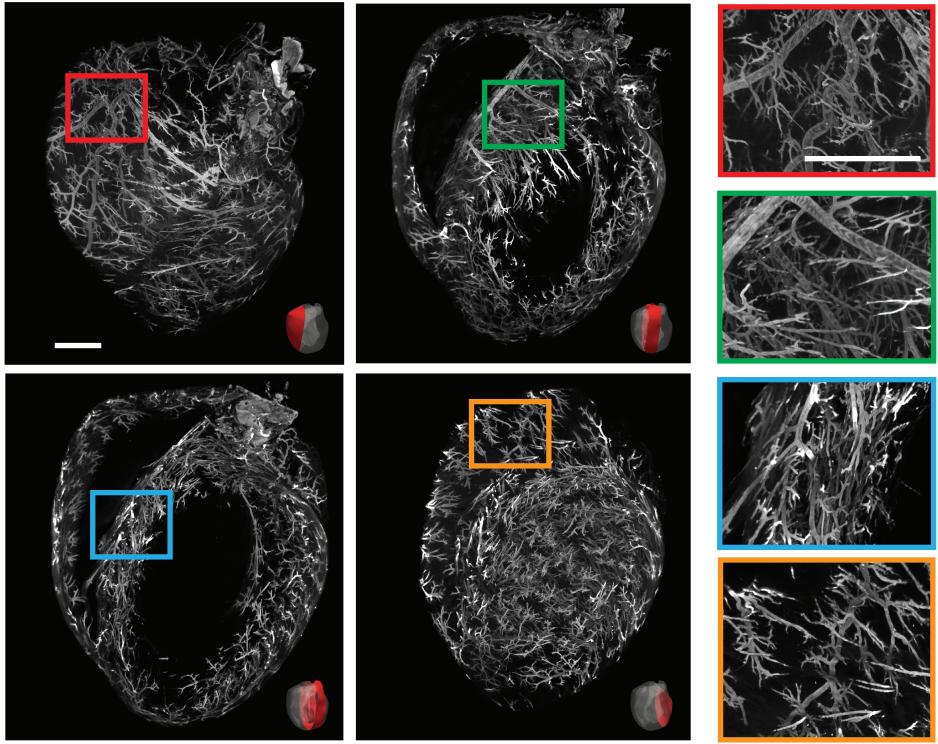

Figure 1: Whole-organ imaging of coronary arteries at cellular resolution. Neonate and adult hearts (atria removed) subjected to tissue clearing and immunolabeling with alpha-smooth muscle actin ( $\alpha-S M A)$ and imaged using a Light sheet microscope. (a) Maximum intensity projection of entire neonate heart. (b) Z-Stack subset projections of Light sheet images $\left(\mathbf{b}_{\text {i-ii }}\right)$ or those captured using a confocal microscope $\left(\mathbf{b}_{\text {iii-v }}\right)$. High magnification of septum shows complexity of the septal artery ( $\left.\mathbf{b}_{\mathrm{iii}}\right)$ and of LCA shows 
colocalization of $\alpha-S M A+$ branches with artery marker Connexin 40 (purple arrowheads)( $\mathbf{b}_{\mathrm{iii}-\mathrm{v}}$ ). An $\alpha-S M \mathrm{~A}^{\text {low }}$ Connexin 40 - vein (orange arrowheads) is also present in $\mathbf{b}_{\text {iii-v. }}$ (c) $3 \mathrm{D}$ rendering of myocardial volume $(\mathbf{r e d})\left(\mathbf{c}_{\mathbf{i}}\right)$ and main coronary artery branches: Right (RCA), Septal (SpA) and Left (LCA)( $\mathbf{c}_{\text {iii). }}$ (d) Maximum intensity projection of entire adult heart. (e and $\mathbf{f})$ Z-Stack subsets of indicated heart regions (e) and region-of-interest $(\mathrm{ROI})$ images (f) reveal the high resolution and specificity of immunolabeling with this technique. Scale bars: a-c, $300 \mu \mathrm{m}$; d-f, $500 \mu \mathrm{m}$.

To characterize collateral arteries using this novel method, we imaged neonatal and adult mice subjected to myocardial infarction (MI)(uninjured mouse hearts do not generally contain collaterals $)^{9,10}$. Collateral arteries form faster in neonates than in adults $^{9}$. Thus, injured neonatal hearts were harvested 4-days post MI while adult hearts were collected 28-days post-MI, followed by arterial immunolabeling and clearing. A collateral tracing pipeline was developed first using images of neonatal hearts (Fig. 2a). ImageJ's Simple Neurite Tracer plugin ${ }^{42,43}$ was used to trace, in a semi-automated way, every a-SMA+ vessel that originated downstream of the LCA occlusion (suture) and connected to either the RCA, SpA, or the LCA upstream of the occlusion. Traced paths were isolated and masked so that 3D rendering in Imaris created a map of every collateral artery found post-MI (Fig. 2b). The resolution of our method allowed us to annotate the precise collateral segments that bridged two artery branches (Fig. $2 \mathrm{~b}_{\mathrm{ii}}$ ). A collateral bridge was defined as the segment of continuous smooth muscle covered vessel that existed between two branch tips with opposing branch angles (Fig. 2c). Tracing did not detect collateral connections in non-injured neonate hearts (Fig. S1). Thus, this method reliably identifies collateral arteries in whole heart images.

To ascertain where collateral bridges were localized with respect to injured myocardium, we labeled all coronary vessels in the neonate with Podocalyxin and used the autofluorescence signal to observe surviving cardiac muscle. Areas lacking

212 myocardium, which was confirmed by accompanying disrupted vasculature (Fig. 2d).

213 Injured regions were outlined and overlaid onto collateral models (Fig. 2 $b_{i}$ ). Collateral

214 bridges were usually located at the edge of the infarcted area, connecting regions of

215 muscle and vascular death to unaffected sites in the heart (Fig. 2d). These same

216 methods were then used to identify collaterals and injured myocardium in adult hearts.

217 Similar patterns were observed (Fig. 2e-g). 
218 We next quantified collateral connection type, numbers, and relative sizes in our 219 images. Collateral connections were categorized based on which artery they connected 220 (Fig. 2h). The majority of connections in both neonate and adult hearts were SpA-LCA. 221 Neonate hearts formed more LCA-LCA and fewer RCA-LCA connections than adults 222 (Fig. 2h). Neonate hearts also formed approximately $40 \%$ more collaterals than adults 223 (Fig. 2i), and their diameters were larger, both absolutely and relative to the proximal 224 LCA (Fig. 2j and data not shown). (Normalization to proximal LCA was performed to 225 account for small differences in individual mouse body size.) These data highlight the 226 importance of advanced imaging methods for observing accurate vascular remodeling 227 patterns, i.e., those involving the septal artery, and underscore the significant 228 differences between young and old hearts. 
Neonate 4 days post-MI
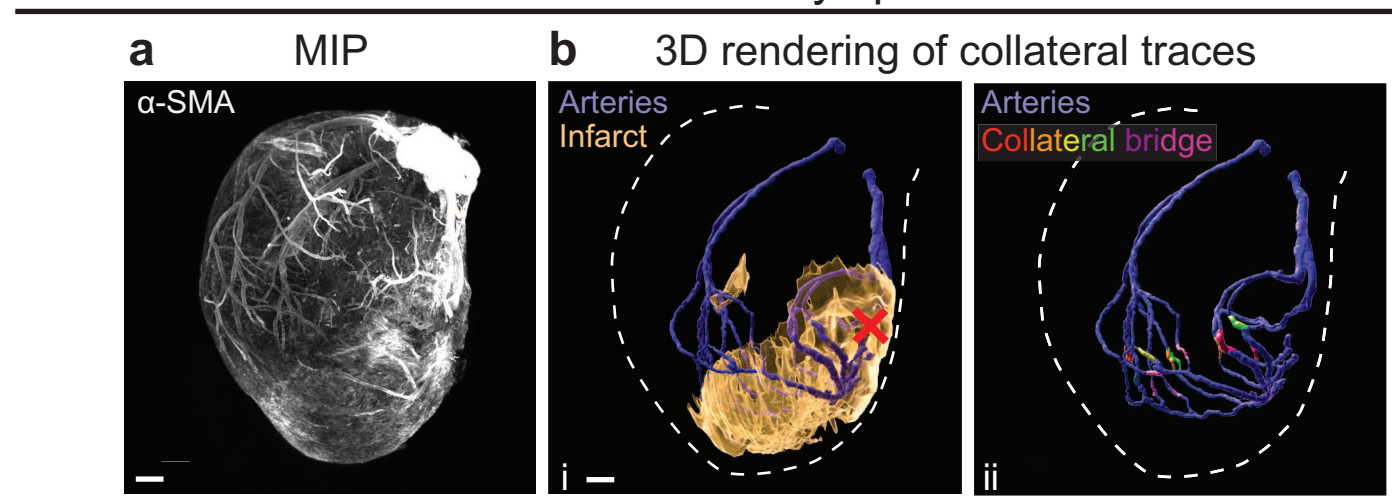

c $3 \mathrm{D}$ reconstruction $\mathbf{d}$

Z-stack subset
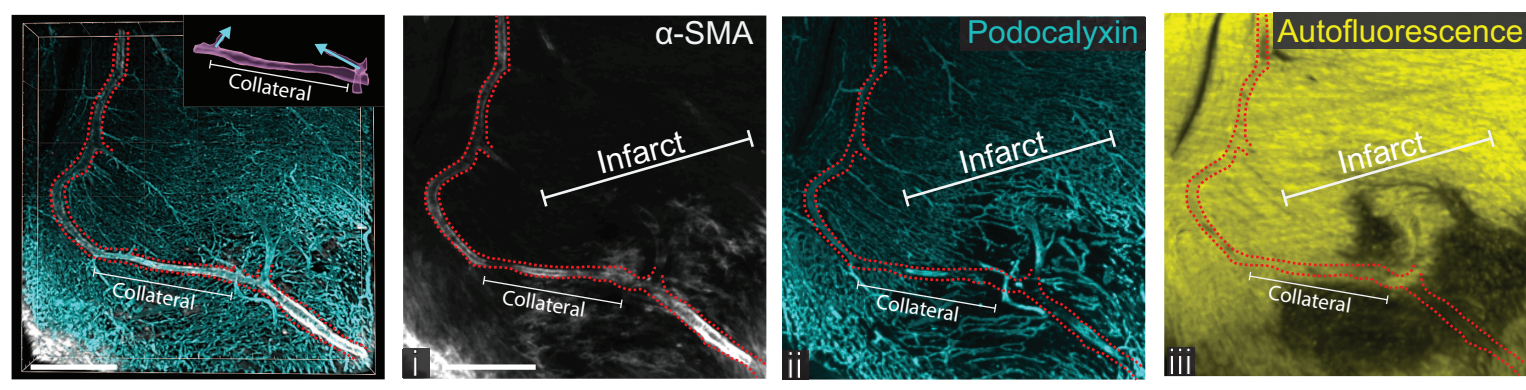

Adult 4 weeks post-MI

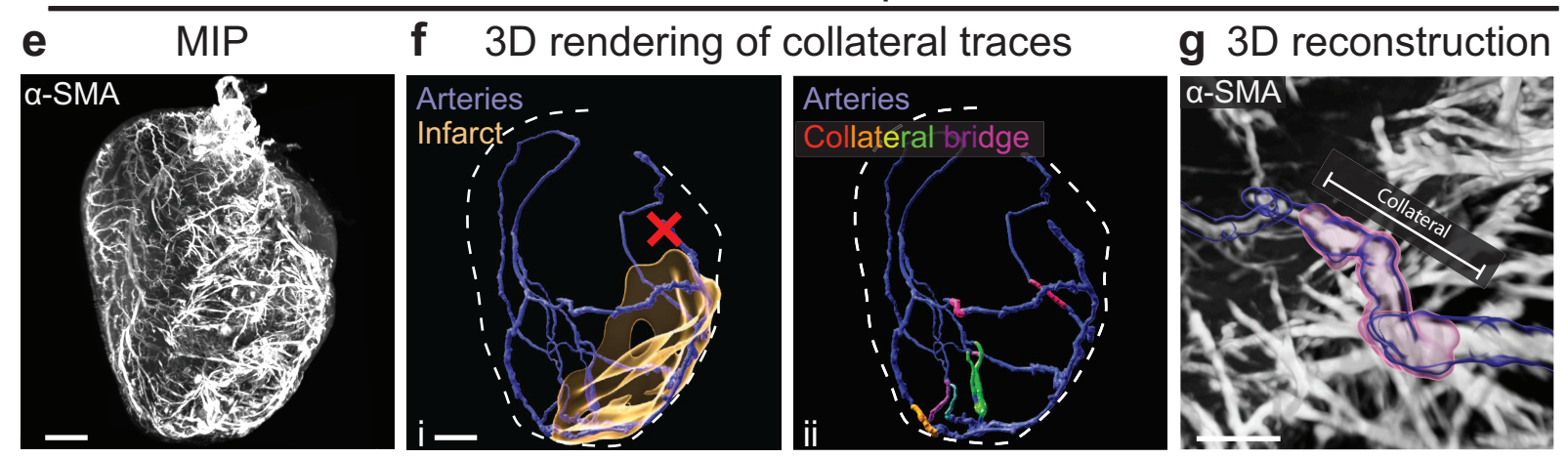

h

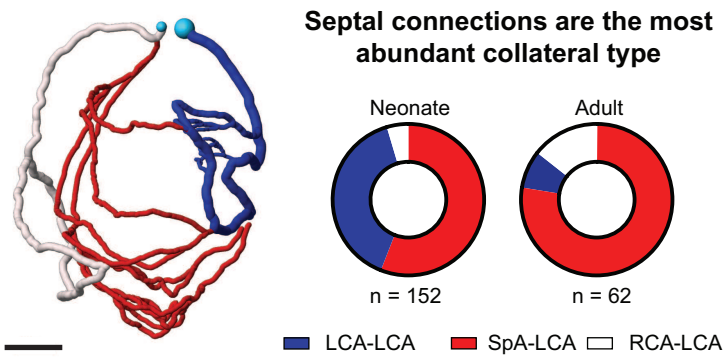

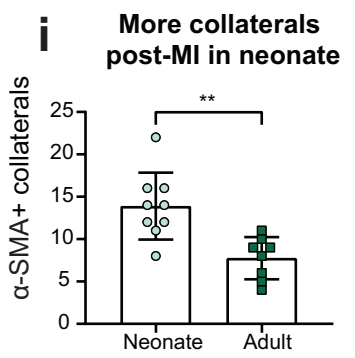

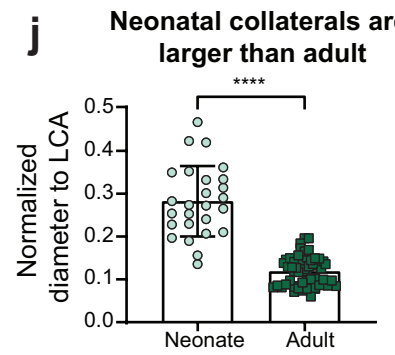

Anbazhakan* and Rios Coronado* et al., Figure 2

230 Figure 2: Increased collateral arteries in neonate versus adult hearts post injury. 231 (a-d) Whole organ imaging of P6 neonatal heart labeled with a-SMA post myocardial 
infarction (MI). (a) Maximum intensity projection (MIP) of entire heart. (b) Collateral connections traced from downstream of suture (red X) were 3D rendered and overlaid with infarct volume $\left(\mathbf{b}_{\mathbf{i}}\right)$ and collateral bridges $\left(\mathbf{b}_{\mathrm{ii}}\right)$. (c) 3D reconstruction of $100 \mu \mathrm{m} \mathrm{Z-}$ stack containing a representative collateral bridge within a traced vessel (red dotted line). (d) MIP of a $35 \mu \mathrm{m}$ Z-stack within c highlighting an $\alpha$-SMA+ collateral $\left(\mathbf{d}_{\mathbf{i}}\right)$ and its relation to Podocalyxin labeling all vessels $\left(\mathbf{d}_{\mathrm{ii}}\right)$ and Autofluorescence labeling surviving myocardium ( $\left.\mathbf{d}_{\mathrm{iii}}\right)$. (e-g) Adult (16-week-old) injured hearts labeled with a-SMA. (e) MIP of entire heart. (f) $3 D$ rendering of collateral connections overlaid with infarct volume $\left(\mathbf{f}_{i}\right)$ or collateral bridges $\left(f_{i i}\right)$. $(\mathbf{g}) 3 \mathrm{D}$ reconstruction of representative collateral bridge (pink). (h) Classification and distribution of collateral connections. (i) Collateral numbers in neonate ( $n=9$ hearts) and adult ( $n=8$ hearts) post-MI. (j) Collateral diameters in neonate $(\mathrm{n}=26$ hearts) and adult ( $\mathrm{n}=55$ hearts) post-MI normalized to the proximal LCA. Scale bars: a-b and e-f, $300 \mu \mathrm{m}$; c-d and g-h, $150 \mu \mathrm{m}$. Right (RCA), left (LCA), and septal $(\mathrm{SpA})$ coronary arteries. Error bars are st dev: ${ }^{* *}, p \leq .01 ;{ }^{* * *}, \mathrm{p} \leq .0001$.

\section{Modeling coronary blood flow}

We next sought to understand how these collaterals might restore blood flow in

248 the presence of a vascular occlusion. An in silico approach was employed that would

249 allow us to computationally estimate blood flow while at the same time manipulating 250 different parameters in isolation, such as collateral number, size, and location. First, an

251 anatomically representative model of the native adult coronary tree was created using

252 the open-source software, SimVascular (www.simvascular.org $)^{44}$, from a Light sheet

253 image of a non-injured adult heart labeled with a-SMA (Fig. 3a). The Light sheet images

254 (Fig. 3ai ) were used as a guide for drawing path lines through every artery in the heart 255 up to tertiary branches (Fig. 3a $a_{i i}$, Methods). Arteries were then segmented by drawing a 256 circle that encompassed the entire width at even intervals along the vessel (Fig. 3a $a_{i i i) .}$

257 SimVascular was used to convert the segmentations into a 3D model (Fig. 3aiv). We 258 next measured the amount of tissue shrinkage that occurs during iDISCO by calculating 259 heart volumes pre- and post-clearing ( $F i g .3 b_{i}$ ). Shrinkage was on average 37\% (Fig. $\left.2603 b_{i i}\right)$, and, thus, the model was computationally uniformly scaled up by 1.58 -fold (Fig.

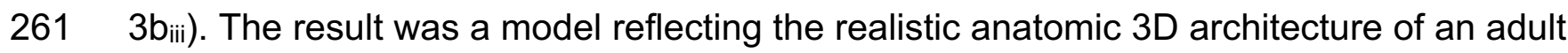
262 mouse coronary artery tree.

263 This model was then used to computationally estimate physiologically realistic

264 blood flow parameters throughout the arterial network. The simulations first required

265 setting boundary conditions. At the aortic inlet, a flow waveform was set based on 266 experimentally measured blood velocities from the literature for the neonate ${ }^{45}$ and 
267 adult ${ }^{46}$. Two outlet boundary conditions were set: 1 . An RCR Windkessel model 268 representing the systemic circulation at the aortic outlet ${ }^{47}$, and 2 . A lumped parameter 269 network (LPN) representing the coronary vessels downstream of the 3D model ${ }^{48,49}$ (Fig. 270 3c). The lumped parameters included values accounting for vessel resistance at 271 downstream arteries, capillaries, and veins and intramyocardial pressure due to 272 contraction of the ventricle (Fig. 3c). Simulations were run on initial estimated 273 parameters (see Methods) and were subsequently tuned to match expected flow splits 274 between coronary branches to ensure our CFD simulation was distributing the flow 275 proportionally. Flow splits were calculated based on perfusion territories for each of the 2763 main branches of the coronary arteries. Each region of the myocardium was 277 connected to its closest arterial end branch, and all the subregions were identified as 278 belonging to branches of either the LCA, RCA, or SpA (Fig. 3 $\mathrm{d}_{\mathrm{i}-\mathrm{ii}}$ ). The method 279 estimated the LCA, RCA, and SpA to perfuse $60 \%, 25 \%$, and $15 \%$ of the myocardium, 280 respectively (Fig. $3 \mathrm{~d}_{\mathrm{ii}}$ ). Using this information to tune outlet boundary conditions (Fig.

281 S2, Methods) resulted in close agreement between estimated perfusion territory and 282 simulated flow splits (Fig. 3d $\mathrm{d}_{\text {iii)}}$. Outlet boundary conditions were held constant as 283 stenosis severity was increased. While this doesn't account for remodeling events due 284 to the stenosis, we are specifically modeling the immediate flow of collaterals arteries 285 that exist before a stenosis would develop. In total, the adjustments to the model and 286 boundary conditions provided a model with close concordance to native physiology. Our next goal was to investigate collateral blood flow, and one benefit of a computational approach is that parameters, such as collateral number/size and stenosis severity, can be virtually modified and systematically tested (Fig. 3e). We placed virtual collaterals within the native coronary tree model described above, using post-injury imaging data to guide general placement (see Fig. 2). Computationally derived pressure 292 values were then used to precisely adjust placement at each branch so that collaterals 293 joined two regions of equal pressure. This minimized flow across collaterals without 294 stenosis, which is important to establish a consistent baseline so that different 295 configurations could be properly compared (Fig. 3e, Fig. S3). These guidelines were 296 used to produce 5 different collateral configurations in the adult heart (Fig. S3). We 297 compared pressure difference, flow, and shear stress in all collaterals from each 
298 configuration to Poiseuille's law, which analytically describes flow through a circular 299 cylinder, to ensure the results of our simulations were reasonable (Fig. S4, Methods). 300 These data verified virtually-placed collaterals for use in computational flow modeling. 301 Because we wanted to make comparisons between adult and neonate hearts, we 302 performed the same workflow with an uninjured P6 heart. Perfusion territories were 303 similar, but a lower aortic inflow was prescribed for neonates to match lower mean 304 pressures following published values ${ }^{45,50}$. Four collateral configurations were produced 305 for neonates (Fig. S3). Then, adult and neonate models were used to investigate re306 perfusion upon virtual stenosis. 

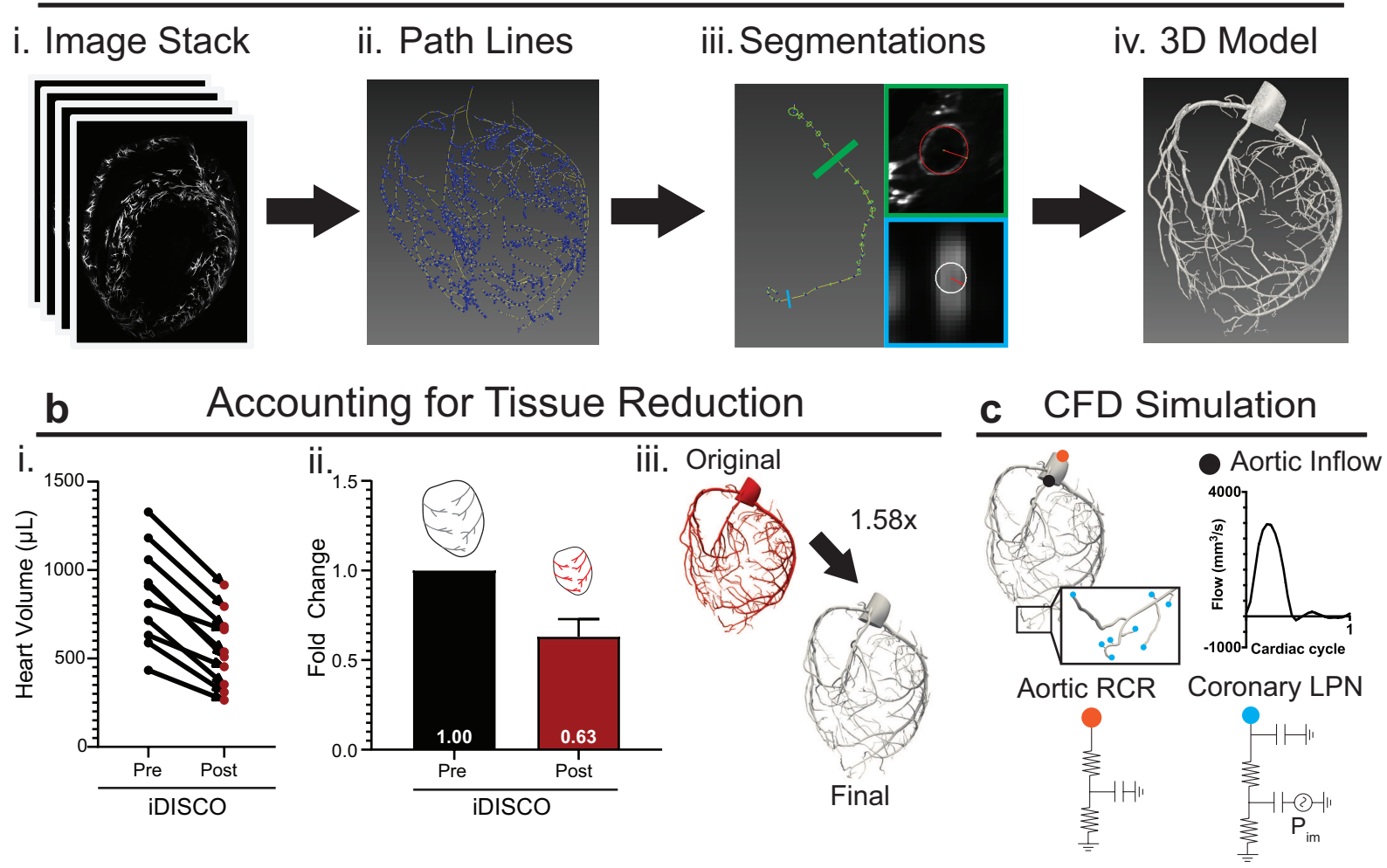

\section{d Perfusion Territories}

i. Individual Outlets ii. Integrated Outlets
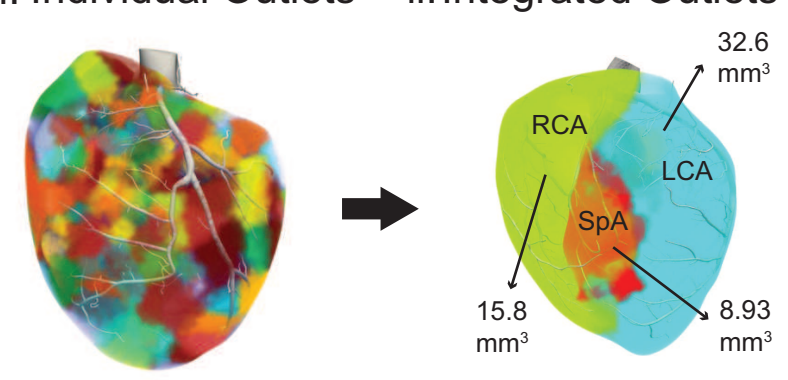

\section{e Virtual Collaterals}

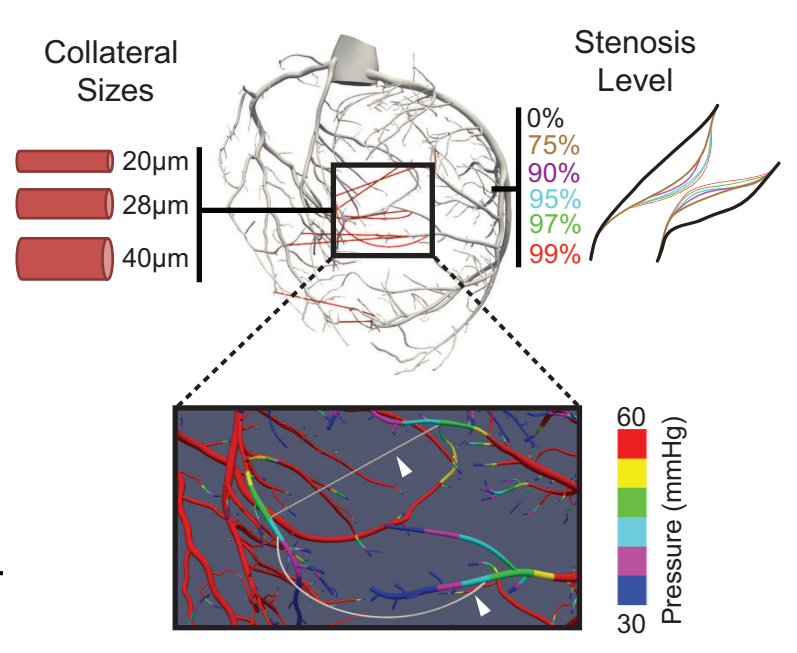

\begin{tabular}{c|cc|cc|c} 
iii. & \multicolumn{2}{|c|}{$\begin{array}{c}\text { Perfusion } \\
\text { Territory }\end{array}$} & \multicolumn{2}{c|}{$\begin{array}{c}\text { CFD } \\
\text { Simulation }\end{array}$} & \multirow{2}{*}{$\begin{array}{c}\text { Percent } \\
\text { Branch }\end{array}$} \\
\cline { 2 - 5 } & $\begin{array}{c}\text { Volume } \\
\left(\mathrm{mm}^{3}\right)\end{array}$ & $\begin{array}{c}\text { Volume } \\
\text { Fraction }\end{array}$ & $\begin{array}{c}\text { Flow } \\
\left(\mathrm{mm}^{3} / \mathrm{s}\right)\end{array}$ & $\begin{array}{c}\text { Flow } \\
\text { Fraction }\end{array}$ & \\
\hline LCA & 32.6 & 0.568 & 5.67 & 0.599 & $5.18 \%$ \\
RCA & 15.8 & 0.272 & 1.7 & 0.254 & $-6.62 \%$ \\
SpA & 8.93 & 0.159 & 0.98 & 0.147 & $-7.54 \%$ \\
Total & 57.3 & 1 & 8.35 & 1 & N/A
\end{tabular}

Anbazhakan* and Rios Coronado* et al., Figure 3

Figure 3: Building a physiologically representative 3D model of mouse coronary arteries. (a) Pipeline for generating 3D models from Light sheet images. (b) Scaling model to account for tissue volume reduction during iDISCO procedure. Measuring heart volumes pre- and post-processing $\left(\mathbf{b}_{\mathbf{i}}\right)$ yielded an average reduction value $\left(\mathbf{b}_{\mathrm{ii}}\right)$ used to generate a scaling factor for models $\left(\mathbf{b}_{\mathrm{iii}}\right)$. (c) Schematic of coronary simulation with a prescribed flow waveform at the inlet, RCR boundary condition at the aortic outlet, and coronary LPN at each coronary outlet. (d) Determining perfusion territories 
315

required first utilizing the Voronoi algorithm to outline perfusion subvolumes for each individual outlet $\left(\mathbf{d}_{\mathbf{i}}\right)$. Then, subvolumes were grouped by right (RCA), left (LCA), and septal $(\mathrm{SpA})$ coronary branches $\left(\mathbf{d}_{\mathrm{ii}}\right)$. Outlet boundary conditions were tuned by matching simulated flow splits to perfusion territories ( $\left.\mathbf{d}_{\mathrm{iii}}\right)$. (e) Schematic depicting variations on collateral and stenosis parameters used in this study. Collaterals were placed to connect approximately equal pressure zones (white arrows). RCR, 3-element Windkessel model; LPN, lumped parameter network; $P_{i m}$, intramyocardial pressure.

\section{Investigating flow recovery by collateral arteries}

We next sought to understand the level of flow recovery by virtual collaterals in the presence of coronary occlusions. One way to quantify re-perfusion is to sum the flows from all outlets downstream of the virtual stenosis and compare this to a normalized baseline flow with no stenosis (set at 100\%). As expected, with no collaterals in adults total flow downstream of stenosis decreases when percent occlusion increases, especially above $90 \%$ (Fig. 4a, top row in chart). When comparing all the configurations tested at all stenosis levels, the configuration with 9 collaterals at $40 \mu \mathrm{m}(9 \mathrm{col}, 40 \mu \mathrm{m})$ provides the most flow recovery benefit, especially at $99 \%$ stenosis where it restores almost $25 \%$ of the non-stenotic flow compared to just $1 \%$ without collaterals (Fig. 4a, right-most column in chart). However, this extent of collateralization does not occur naturally with coronary artery ligation in adult mice (see Fig. $2 \mathrm{i}$ and refs $9,10)$. We noted that configurations similar to those observed experimentally, i.e. 6$12 \mathrm{col}, 20 \mu \mathrm{m}$, recovered very little flow as measured by this method (Fig. 4a). These data demonstrate that collateral arteries as they naturally form after adult coronary occlusion are not expected to appreciably recover blood flow, but that increasing diameters, which is a major factor in reducing overall resistance, could enhance their function.

In contrast to the poor function of adult collaterals, those of the same size and number in neonates performed well. The configuration that naturally forms in neonates (i.e. $12 \mathrm{col}, 20 \mu \mathrm{m}$, see Fig. $2 \mathrm{i}$ and ref 9 ) is estimated to recover up to $60 \%$ of total flow downstream of a $99 \%$ stenosis (Fig. 4b). Remarkably, the largest diameter tested (40 $\mu \mathrm{m}$ ) only required one vessel to provide massive recovery in neonates (Fig. 4b, last row in chart). As mentioned above, to compare adult and neonate flow recoveries, it is important to confirm that collaterals generally connect equal pressure zones $(+/-10$ 
$347 \mathrm{mmHg}$ ) so that all configurations start with a similar collateral flow. This was further 348 evident by the observation that adding collaterals did not change total downstream flow 349 without stenosis and primarily increased flow only with increasing stenosis severity (Fig. 350 S3). We concluded that collaterals in neonate hearts perform better than in adults. 351 The above analysis calculated overall recovery of pre-stenosis levels, but clinical 352 data indicate that myocardial tissue could be supported at approximately $30 \%$ of 353 baseline flow ${ }^{51,52}$. Thus, we next sought to gain a more nuanced understanding of 354 recovery by considering individual outlet perfusion territories downstream of the 355 stenosis, so that we could observe if certain regions were receiving sustainable re356 perfusion (i.e. $>30 \%$ re-perfusion). First, we grouped all perfusion territories downstream 357 of the stenosis to obtain the full volume-at-risk (Fig. 4c) and then plotted the percentage 358 of that volume that is re-perfused above a certain threshold (Fig. $4 \mathrm{c}_{\mathrm{i}}$ ). This revealed that 359 while there were still no sustainably re-perfused regions in the $12 \mathrm{col}, 20 \mu \mathrm{m}$ and $6 \mathrm{col}$, $36028 \mu \mathrm{m}$ configurations, the $3 \mathrm{col}, 40 \mu \mathrm{m}$ and $9 \mathrm{col}, 40 \mu \mathrm{m}$ configurations were able to sustain

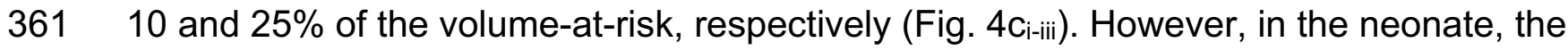
$3626 \mathrm{col}, 20 \mu \mathrm{m}$ re-perfused $80 \%$ of the volume-at-risk over the $30 \%$ threshold while the $36312 \mathrm{col}, 20 \mu \mathrm{m}$ and $1 \mathrm{col}, 40 \mu \mathrm{m}$ configurations re-perfused the entire volume-at-risk (Fig. $3644 \mathrm{~d}_{\mathrm{i}-\mathrm{iii}}$. These data emphasized that collateral configurations of the same size, and thus 365 same resistance, function better in the neonatal heart. 


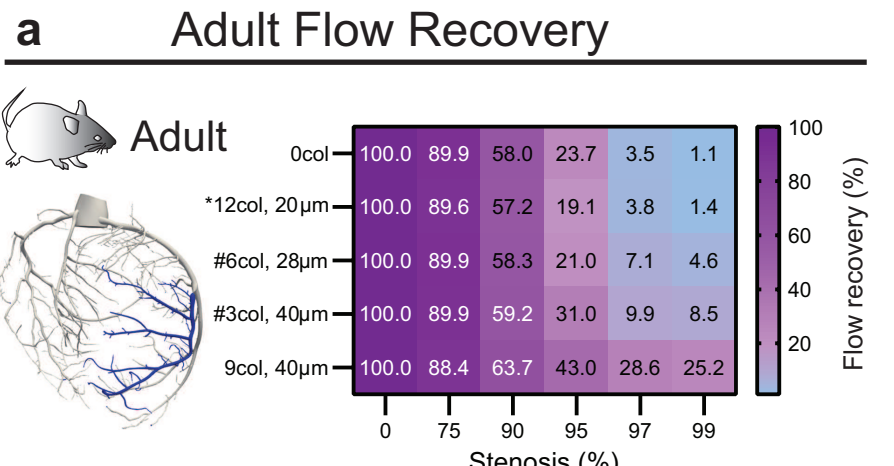

Stenosis (\%)

\section{b Neonate Flow Recovery}
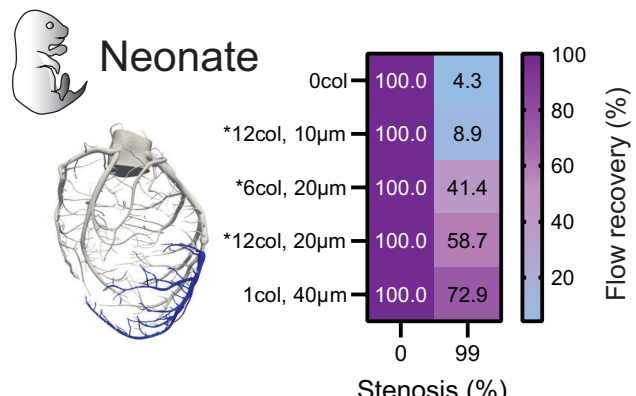

Stenosis $(\%)$

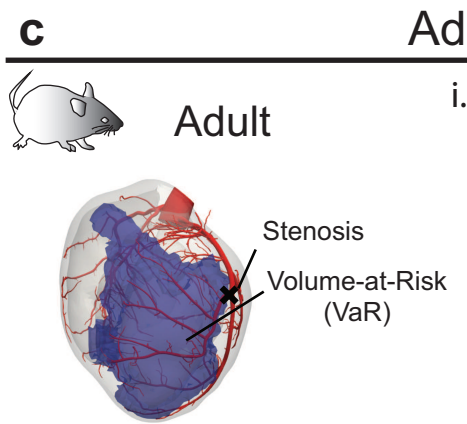

Adult Volume-at-Risk Re-perfused

ii.

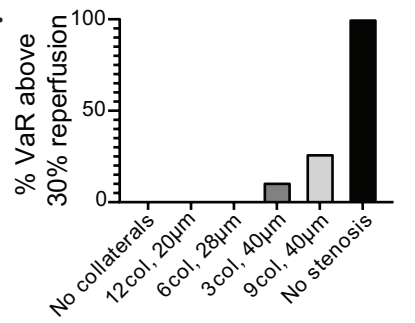

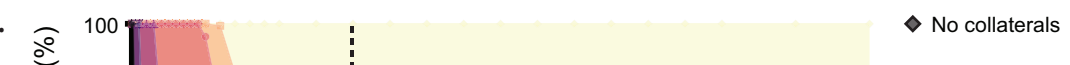

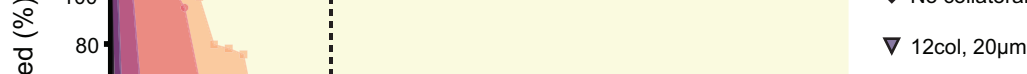

$\Delta 6 \mathrm{col}, 28 \mu \mathrm{m}$

○ $3 \mathrm{col}, 40 \mu \mathrm{m}$

$\square$ 9col, $40 \mu \mathrm{m}$

d

Neonate Volume-at-Risk Re-perfused

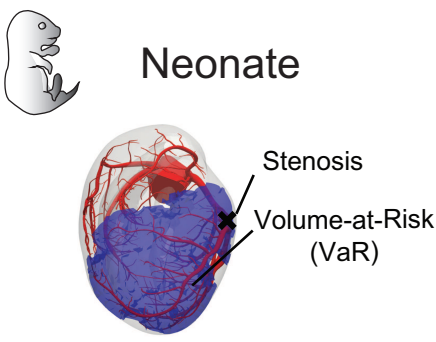

ii.

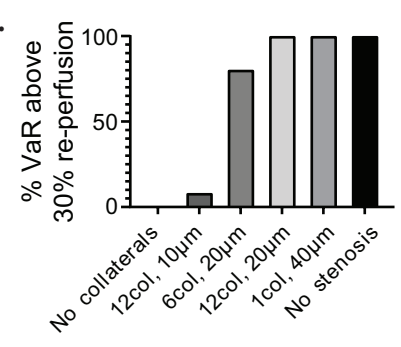

iii.



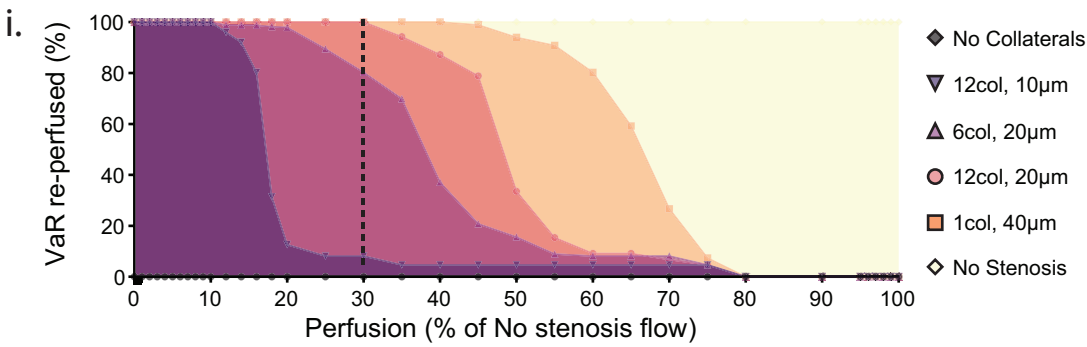

iii.
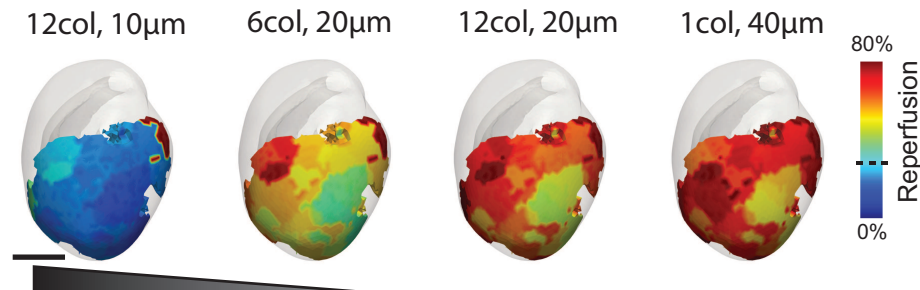
Figure 4: Collateral arteries are predicted to perform better in neonate hearts. (a and b) Measuring re-perfusion capacity by calculating percent of total non-stenotic flow in vessels downstream of the virtual occlusion (blue vessels). Asterisks denote configurations observed experimentally; hashtags denote sizes observed following CXCL12 injection ${ }^{9}$. Functionally significant re-perfusion is only seen in neonates under physiological conditions. (c and d) Percent re-perfusion of myocardial volume-at-risk (VaR)(blue region) in adult (c) and neonatal (d) models. Cumulative histogram $\left(\mathbf{c}_{\mathbf{i}}, \mathbf{d}_{\mathbf{i}}\right)$, bar graph of percent $\mathrm{VaRs}$ above $30 \%\left(\mathbf{c}_{\mathrm{ii}}, \mathbf{d}_{\mathrm{ii}}\right)$, and visual maps of re-perfusion within the $\operatorname{VaR}\left(\mathbf{c}_{\mathrm{iii}}, \mathbf{d}_{\mathrm{iii}}\right)$. Dotted line marks the ischemia threshold of $30 \%$ non-stenotic flow. Scale bar: $1000 \mu \mathrm{m}$.

We next explored whether a more favorable placement of collaterals could

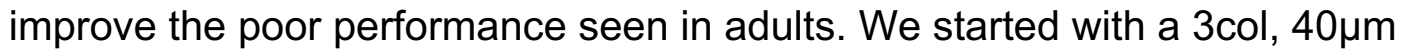
configuration (Fig. 5a, blue) and moved each collateral to a more proximal location in the coronary tree (Fig. 5a, red). This manipulation almost doubled total flow recovery (Fig. 5b) and approximately tripled the volume of myocardium re-perfused above the $30 \%$ threshold (Fig. 5c-e). Thus, variation in location can improve collateral function, likely because the pressure difference with more proximal attachments is much greater, resulting in increased flows.

The above data suggested that fewer, larger collaterals are better than many, smaller ones (see Fig. 4). However, in those experiments, the total collateral resistance varied between the configurations. We tested this hypothesis by varying the number and size of the collaterals while keeping the total resistance equal. Simulations were performed on 2 configurations-16col, $20 \mu \mathrm{m}$ and $1 \mathrm{col}, 40 \mu \mathrm{m}$ (Fig. 5f). While total flow recovery was approximately equivalent (Fig. $5 \mathrm{~g}$ ), the $1 \mathrm{col}, 40 \mu \mathrm{m}$ configuration was uniquely able to re-perfuse $5 \%$ of the volume-at-risk above $30 \%$ (Fig. $5 \mathrm{~h}-\mathrm{j}$ ). This analysis shows that fewer, larger collaterals could be more beneficial because they at least protect a portion of the myocardium while many, smaller collaterals distribute the re-perfusion so that none reach protective levels. 
Placement of collateral affects flow

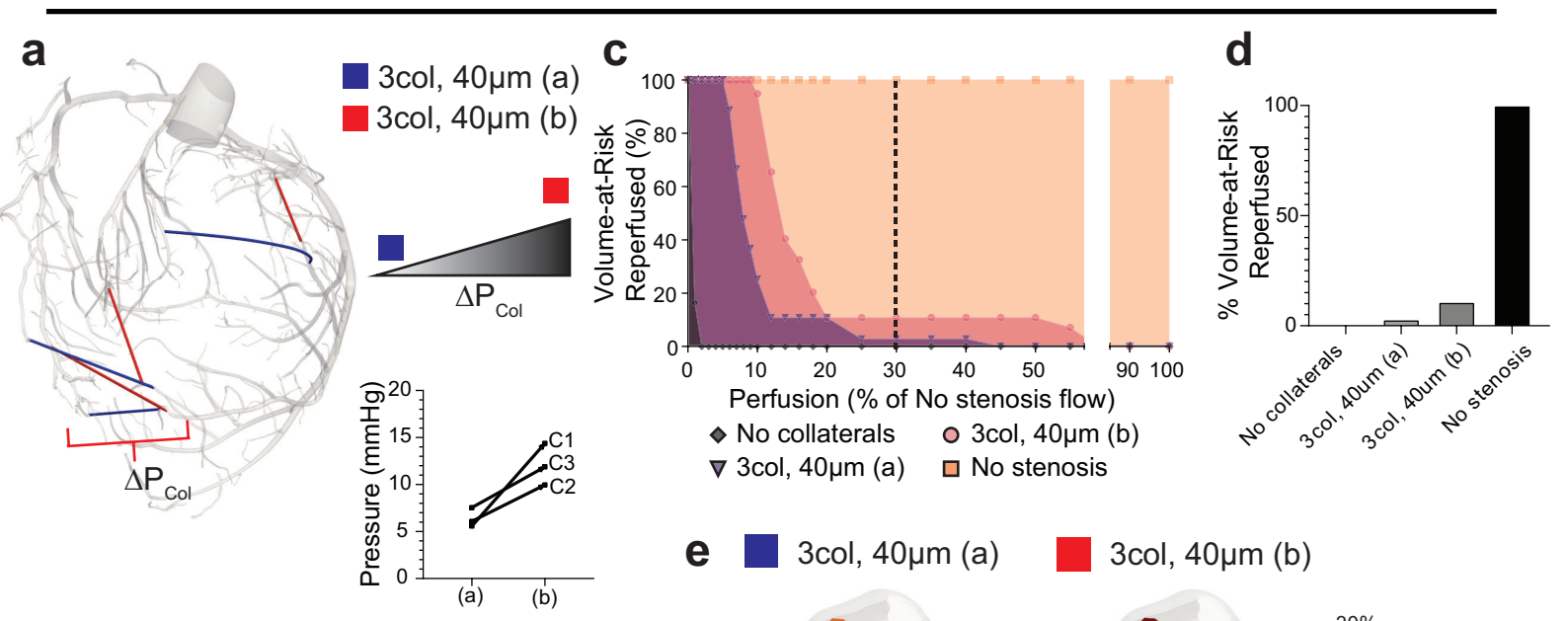

b


Equal Total Collateral Resistance

Tradeoff between many and few collaterals

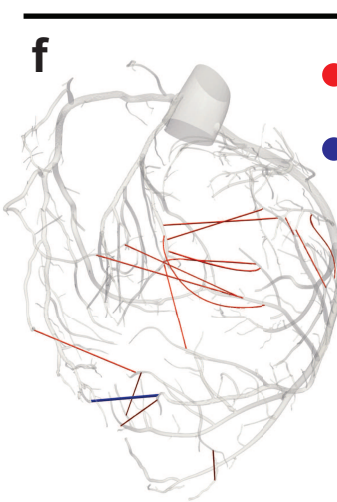

g

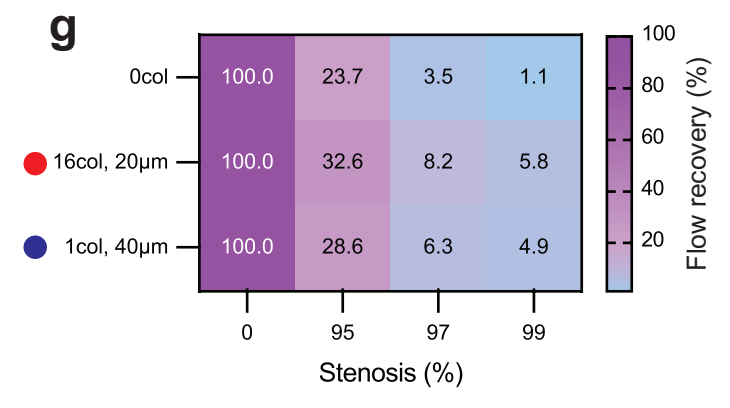

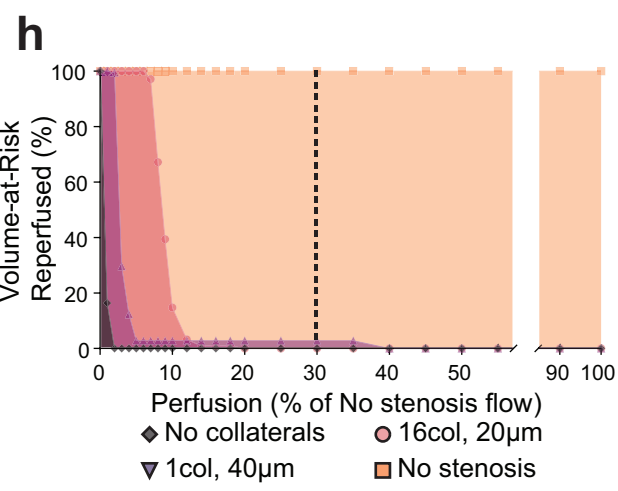

i

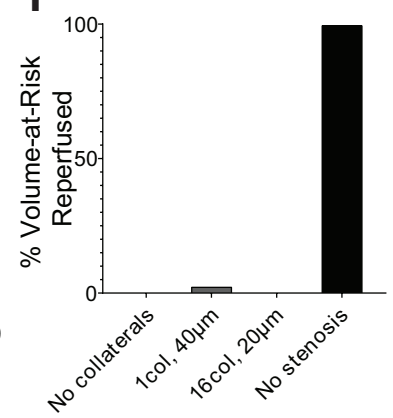

$1 \mathrm{col}, 40 \mu \mathrm{m}$

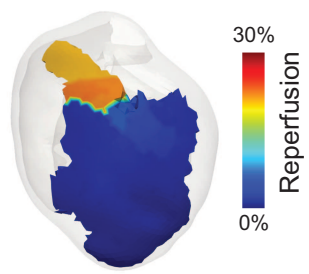

Equal Total Collateral Resistance

Anbazhakan* and Rios Coronado* et al., Figure 5 
400 using two collateral configurations. (a) 3 collaterals with either high (red square) or low $401 \Delta P_{C o l}$ (blue square). (a) Graph showing the $\Delta P_{\text {Col }}$ changes caused by altering placement 402 for each collateral. ( $\mathbf{f}-\mathbf{j})$ Investigating re-perfusion tradeoff between many, small and few, 403 large collaterals using two configurations: 16 collaterals at $20 \mu \mathrm{m}$ (red circle) and 1 collateral at $40 \mu \mathrm{m}$ (blue circle). (b, g) The total non-stenotic flow in vessels downstream of the virtual occlusion. Cumulative histogram $(\mathbf{c}, \mathbf{h})$, bar graph of percent volume-at-risk $(\mathrm{VaR})$ above $30 \%(\mathbf{d}, \mathbf{i})$, and visual maps of re-perfusion within the $\mathrm{VaR}(\mathbf{e}, \mathbf{j})$. Dotted line marks the ischemia threshold of $30 \%$ non-stenotic flow. $\Delta P_{C o l}$, pressure difference across collaterals.

\section{Adult vs. neonate coronary artery morphology}

Given that collaterals of the same size and total resistance were predicted to

411 proportionally recover more flow in the neonate, we sought to understand why and first

412 investigated arterial pressures at collateral formation sites at both ages. To facilitate

413 comparisons between the two time points, Strahler ordering was used to classify branch

414 segments into orders based on hierarchal position in the coronary tree and vessel

415 diameter ${ }^{53,54}$. Order 13 represented the aorta, order 12 represented the most proximal

416 coronary artery segments, and subsequent orders represented downstream vessels

417 until 8, which were the most distal branches modeled (Fig. 6a). This was used to

418 compare hemodynamic and anatomical quantities at similar points in the coronary tree

419 in both the adult and neonate. While absolute aortic and proximal coronary (order 12)

420 pressures were vastly increased in the adult, the pressures at the most distal coronary

421 tips (order 8) were approximately equal (Fig. 6b and c). Quantification revealed that the

422 pressure drop along the coronary tree was $\sim 20$ and $\sim 50 \mathrm{mmHg}$ in neonate and adults,

423 respectively (Fig. 6c). This is also true when considering just the segments downstream

424 of the stenosis, making $\Delta \mathrm{P}_{\mathrm{Ad}}>\Delta \mathrm{P}_{\mathrm{Neo}}$ (Fig. $6 \mathrm{~b}$ and $\mathrm{c}$ ). Thus, the collateral pressure

425 difference $\left(\Delta \mathrm{P}_{\mathrm{Col}}\right)$ required to restore pre-stenotic flow downstream of the occlusion is

426 higher in the adult. Specifically, the $\Delta \mathrm{P}_{\text {col }}$ needs to be about $\sim 2$-fold more in the adult to

427 restore the same flow. Given that we see similar distal pressures at both stages, this

428 explains why, even though collaterals in both recover the same absolute flow, it is much

429 lower than the baseline, non-stenotic flow in the adult.

430 Our next experiments were aimed at understanding why $\Delta \mathrm{P}_{\mathrm{Ad}}$ was greater than

$431 \Delta \mathrm{P}_{\text {Neo. }}$. Two factors critical for determining $\Delta \mathrm{P}$ are flow rate and total resistance of the

432 coronary tree. First, we compare the flow rate at each Strahler order between the 
433 neonate and the adult coronary models. Literature values indicated that aortic flow in 434 adults is approximately ten times more than neonate, which was used as the inflow 435 boundary condition for the computational model (see Fig. 3c) ${ }^{45}$. Simulations revealed 436 that flow was also 10 -fold greater for every vessel order modeled in the coronary tree 437 (Fig. 6d). Shear stress was lower in neonates compared to adults, particularly in higher 438 order vessels (Fig. 6e). We confirmed this trend held true when increasing the mesh 439 size from 1.8 to 10 million elements; there was less than $10 \%$ difference in average 440 shear stress with increased mesh resolution. Flow values were in line with increases in 441 myocardial volume over time, i.e. volumes at P32 were more than 10-fold of P0 (Fig. 6f).

442 The ability to rapidly determine volumes allowed us to analyze additional timepoints, 443 which revealed a linear increase in myocardial volume during the first two weeks of life, 444 a plateau between P18-25, and a burst of growth from P25-32. Second, we used the 445 simulated flows and pressures to calculate the total resistance of the 3D coronary 446 model. Neonate total vascular resistance was 3-fold that of adults (Fig. $6 \mathrm{~g}$ ). Since flow 447 was increased by 10 -fold, the 3-fold decrease in total resistance is not enough to offset 448 flow increases. Thus, while the resistance of the coronary vasculature decreases in the 449 adult, it's not able to lower the resistance enough to balance the much greater flow, 450 which manifests in a greater $\Delta \mathrm{P}$ in adults. 

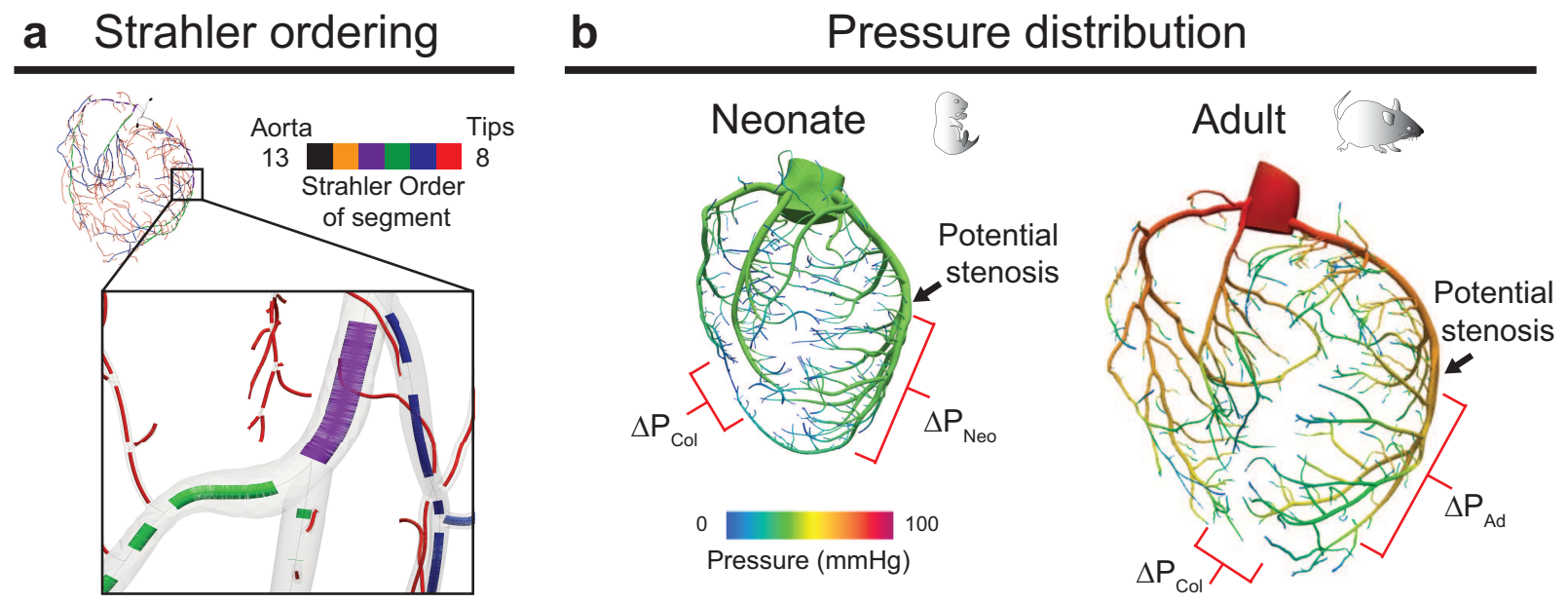

Greater pressure loss due to increased flow
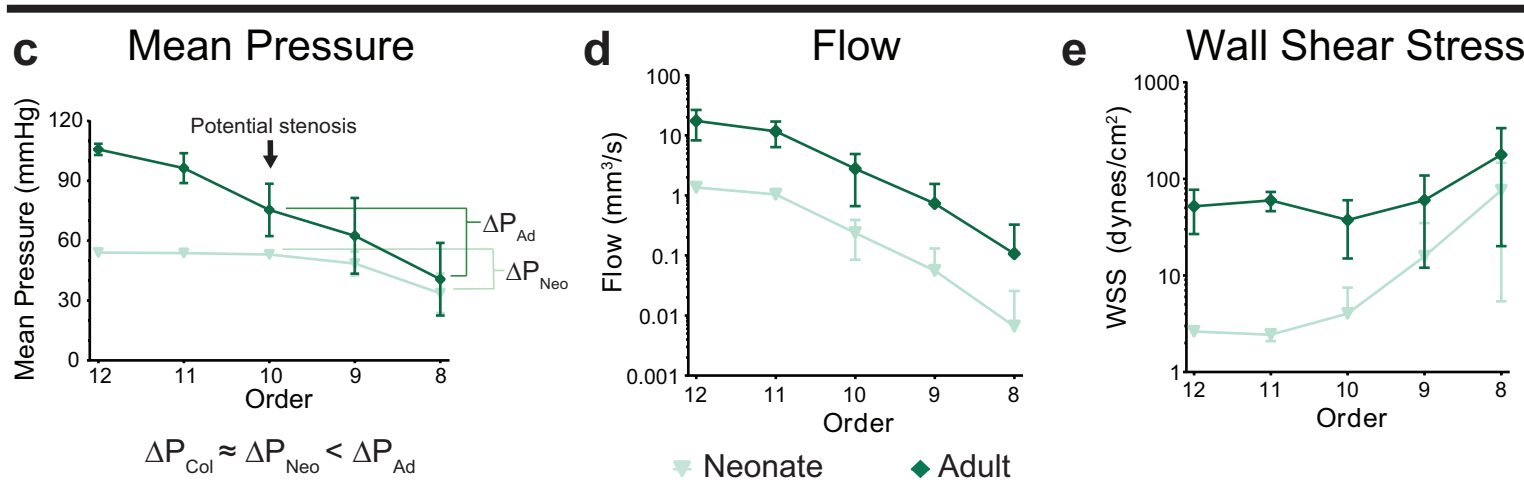

f Heart volume increases proportional to flow



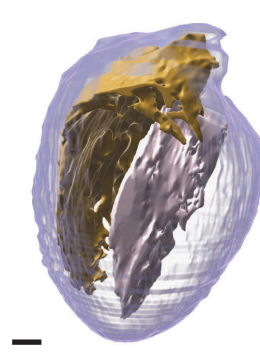

-



Anbazhakan* and Rios Coronado* et al., Figure 6

Figure 6: Investigating hemodynamic differences between neonate and adult. (a) 8 (distal tips). (b) Pressure distribution in the neonate and adult coronary models. (c-e) Quantification of pressure (c), flow (d), and wall shear stress (e) vs. Strahler order $(n=1$ P6, $n=1$ P60 heart model). (f) Heart volume segmentation (left) and quantification ( $n=3$ P0, n=7 P6, n=3 P11, n=2 P18, n=2 P25, n=2 P32 hearts). (g) Total 3D resistance of the coronary tree in neonate and the adult models revealed a 3-fold decrease in adults. $\Delta P_{C o l}$, pressure difference across collaterals; pressure difference downstream of a 
potential stenosis in the neonate, $\Delta P_{N e o}$, and the adult, $\Delta P_{A d}$. Error bars are st dev: ${ }^{* *}$, $\mathrm{p} \leq .01 ;{ }^{* * *}, \mathrm{p} \leq .001 ;{ }^{* * * *}, \mathrm{p} \leq .0001$.

We next investigated what features contribute to the nonproportional decrease in resistance with respect to the flow increase from neonate to adult. Two factors critical for determining resistance are vessel diameter and number of branches. Increases in these parameters both work to lower total resistance, diameter being the most impactful. Surprisingly, we found that the diameters were the same across all Strahler orders in each model (Fig. 7a). We validated this by comparing diameters of the most proximal segments of the RCA, SpA, LCA and aorta in multiple replicates of neonatal and adult hearts (Fig. 7b). The coronary stem diameter remains virtually the same while aortic diameter increased with age (Fig. 7b), a result we validated using an orthogonal method (Fig. 7c). Thus, coronary diameters do not grow proportionally to heart volume, which suggests that diameter expansion does not function to relieve vascular resistance in the face of increased flow demand in adults.

If arteries do not increase in diameter, additional branches must be added to at least partially offset the increased flow that accompanies heart growth. We next quantified branching during postnatal development. Comparing the Strahler ordering of the two stages revealed that the number of distal vessels (order 9 and 8 ) were vastly increased (Fig. 7d), aligning with qualitative observations from imaging (see Fig. 1). Since the 3D SimVascular models did not contain arterioles distal to tertiary branches, we further investigated morphometry by manually tracing all $\alpha$-SMA vessels in a representative branch-the Left Circumflex (LCx)(Fig. 7e, red). Imaris software filament

483 tracing binned each segment of the LCx according to branching levels and quantified 484 the number of arteriole tips (Fig. 7f). The number of branching levels spiked between 485 P0-6 and then hit a plateau until another spike between P25-32 (Fig. 7g). Number of tips increased linearly up to P18 with another spike between P25-32 (Fig. 7h). The P6-

48718 plateau in number of branch levels compared to the linear increase in number of tips 488 over the same time period indicated that the coronary arteries grow by adding branch 489 segments along the entire length of existing branches. We also observed that the length 490 of each segment was constant among all ages tested (Fig 7i). This results in a coronary 491 tree with many lateral branch segments of a set length. 
Coronary diameters remain constant


Branching and number of tips increase

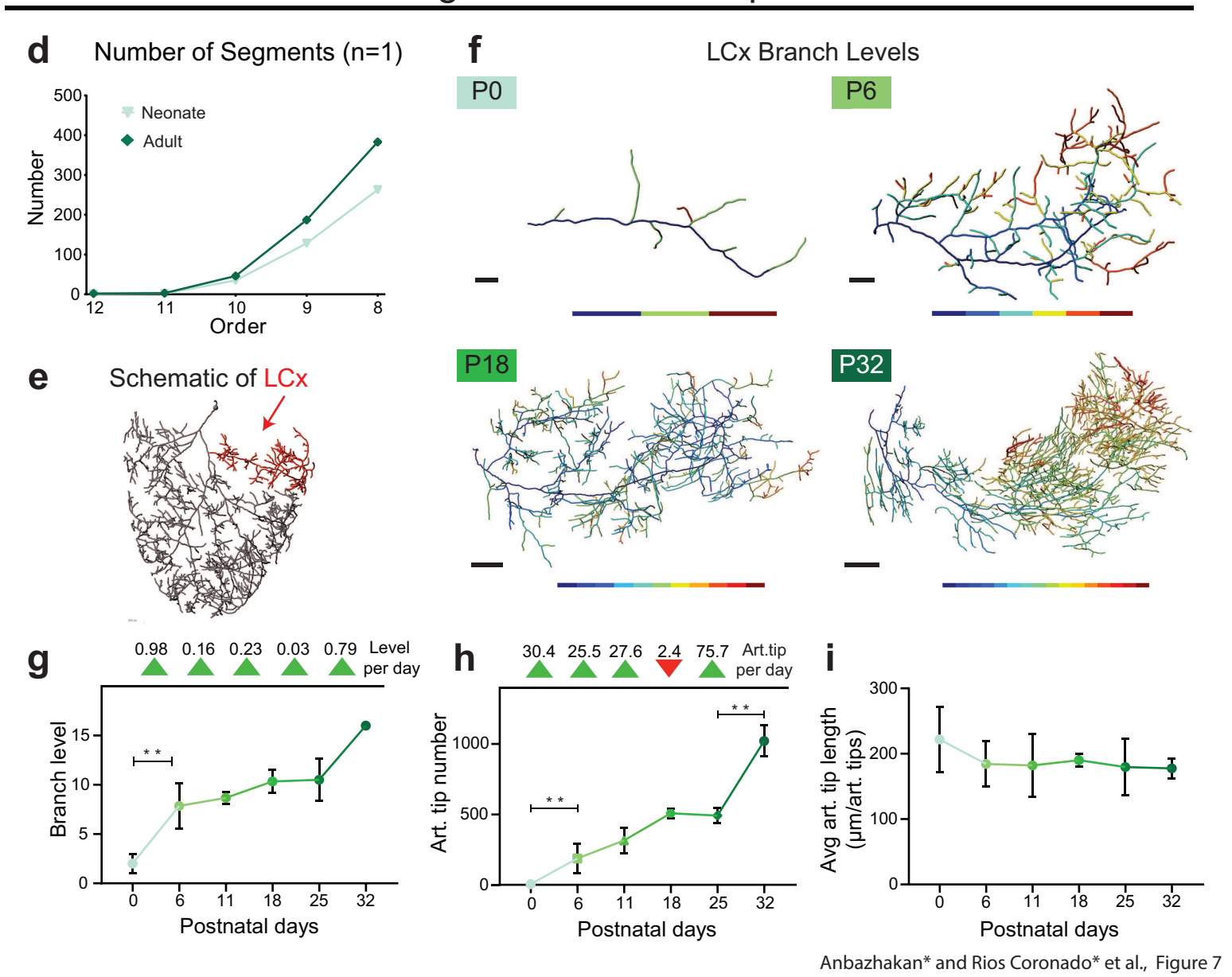

494 Figure 7: Main branch coronary artery diameters remain constant while branching increases throughout postnatal development. (a) Quantification of diameter vs. Strahler order. (b and c) Main branch coronary diameter measurements from additional hearts processed through iDISCO (b) or conventional whole mount immunostaining without clearing (c). (d) Number of artery segments per Strahler order. 
501 Number of branch levels of LCx at each timepoint. Arrowheads indicate rate of change 502 per day. (h) Number of arterial (art.) tips in the LCx at each age. Arrowheads indicate 503 rate of change per day. (i) Average art. segment length in LCx at each timepoint. (a,d) $504 \mathrm{n}=1$ P6, n=1 P60 hearts; (b) $n=4$ P6, n=3 P25 hearts; (c) n=5 P6, n= 4 P60 hearts; $505(\mathbf{g}, \mathbf{h}, \mathbf{i}) \mathrm{n}=3 \mathrm{P0}, \mathrm{n}=7 \mathrm{P} 6, \mathrm{n}=3 \mathrm{P} 11, \mathrm{n}=2 \mathrm{P} 18, \mathrm{n}=2 \mathrm{P} 25, \mathrm{n}=2 \mathrm{P} 32$ hearts. Scale bars: $\mathbf{f}, \mathrm{P0}$, $506100 \mu \mathrm{m} ; \mathrm{P} 6,200 \mu \mathrm{m} ; \mathrm{P} 18,400 \mu \mathrm{m} ; \mathrm{P} 32,500 \mu \mathrm{m}$. Error bars are st dev: ${ }^{*}, \mathrm{p} \leq .01$.

\section{Human fetal and adult coronary collateral arteries}

508

509

510

511

512

513

514

515

516

517

518

519

520

521

522

523

524

525

526

527

528

529

530

531

532

A subset of human hearts contains collateral arteries, which are easily observed during an angiogram and are correlated with increased survival in heart disease patients ${ }^{55,56}$. We sought to identify how our computational modeling studies could help us better understand human collateral function. Thus, we compared the data available from human hearts to our mouse models. We measured vessel diameters for the collaterals observable in angiograms from five patients living with chronic total occlusions (Fig. 8a). This patient population was chosen because their collaterals would be expected to sufficiently support myocardial perfusion downstream of the occlusion without exercise. To compare to mouse data, we also normalized human diameters to the most proximal segment of the LCA. Collateral diameters were on average 15 percent of the LCA (Table 1). These values were in between those observed in the neonate and adult mouse hearts (Table 1). However, a limitation of this comparison is that diameters in angiograms were measured in a 2D projection, which may affect accuracy of absolute values. We also found an average of 2 collaterals per heart (Table 1 ), but comparisons with mouse data using this parameter are less desirable because angiograms will only highlight a subset of the collaterals that immunostaining would label. These data provide a foundation to determine re-perfusion benefit, but a very precise understanding in humans will need to consider the different pressure distributions resulting from human specific morphology.

Using post-mortem perfusions, studies from the 1960s reported the presence of coronary collateral arteries in infants and children ${ }^{57,58}$, but no one has reported whether collaterals develop during embryogenesis. Furthermore, using smooth muscle coverage to identify collateral connections in humans has not been done. We processed two fetal hearts aged 17 and 22 weeks with the same whole-organ immunolabeling method used for murine hearts (Fig. 8b $b_{i-i i}$ and Fig. S5). Both hearts had visible collaterals on the 
533 dorsal and ventral sides (Fig. $8 \mathrm{~b}_{\mathrm{iii} i \mathrm{iv}}$ and Fig. S5). Remarkably, $>17$ collaterals were 534 detected per side (Fig. 8c), which suggests that the whole human heart has at least 40 535 pre-existing, smooth muscle covered collaterals forming during embryonic development. 536 On the ventral side, most connections bridged distal branches of the RCA and LCA 537 while the majority on the dorsal side connected two LCA branches (Fig. 8d). Collateral 538 diameters were not significantly different across locations or between ages and were on 539 average 7 percent of the most proximal LCA segment (Fig. 8e). Thus, unlike mouse, 540 human hearts have mechanisms in place to form native collateral arteries as part of 541 normal development, which we hypothesize could be the precursors for those that 542 preserve myocardium downstream of an occlusion. 

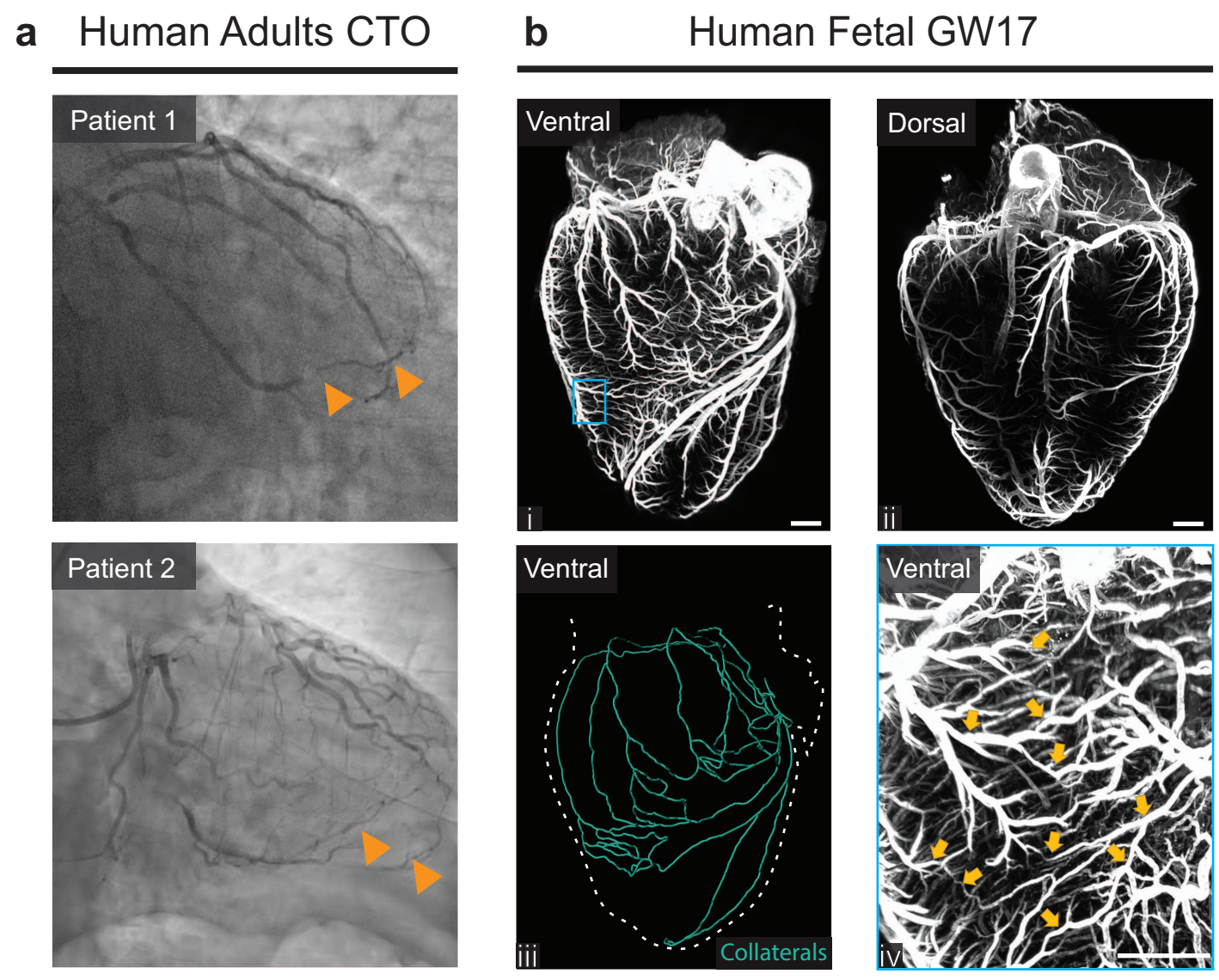

Quantification Human Fetal GW17 and GW22

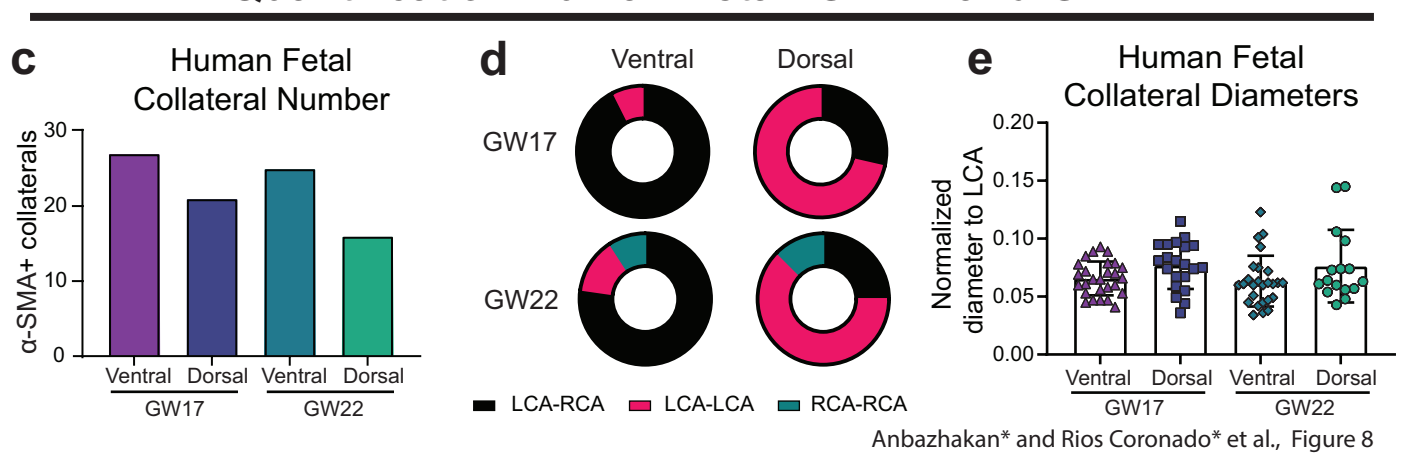

544 Figure 8: Collateral arteries in adult and fetal human hearts. (a) Representative 545 invasive angiograms from adult chronic total occlusion (CTO) patients. Orange 546 arrowheads indicate collaterals. ( $\left.\mathbf{b}_{\mathrm{i}-\mathrm{ii}}\right)$ Maximum intensity projections of fetal human 547 heart, ventral $\left(\mathbf{b}_{\mathbf{i}}\right)$ and dorsal $\left(\mathbf{b}_{\mathrm{i}-\mathrm{ii}}\right)$ sides. $\left(\mathbf{b}_{\mathrm{iii}}\right)$ Traced collateral connections on the 548 ventral side. $\left(\mathbf{b}_{\mathrm{iv}}\right)$ High magnification (boxed in $\mathbf{b}_{\mathbf{i}}$ ) of collateral bridges (arrowheads). (c549 e) Quantification of collateral bridge numbers (c), connection types (d), and diameters 550 551 


\begin{tabular}{|l|l|l|l|l|l|l|}
\hline Species & Age & Condition & $\begin{array}{c}\text { Number of } \\
\text { Collaterals }\end{array}$ & $\begin{array}{c}\text { Diameter } \\
\text { Ratio to LCA }\end{array}$ & $\begin{array}{c}\text { LCA } \\
\text { Diameter } \\
(\mathbf{m m})\end{array}$ & Measurement \\
\hline \multirow{3}{*}{ Human } & Adult & CTO & $1.80 \pm 0.84$ & $0.15 \pm 0.06$ & $5.67 \pm 1.09$ & Angiogram \\
\cline { 2 - 7 } & Fetal GW17 & Native & 48 & $0.07 \pm 0.02$ & 0.42 & LSM \\
\cline { 2 - 7 } & Fetal GW22 & Native & 43 & $0.07 \pm 0.03$ & 0.53 & LSM \\
\hline \multirow{2}{*}{ Mouse } & Neonate & Ml-4 days & $13.9 \pm 3.95$ & $0.28 \pm 0.08$ & $0.12 \pm 0.04$ & LSM \\
\cline { 2 - 7 } & Adult & Ml-4 weeks & $7.75 \pm 2.49$ & $0.12 \pm 0.03$ & $0.13 \pm 0.04$ & LSM \\
\hline
\end{tabular}

Table 1. Human fetal hearts have many relatively small collateral arteries. GW17 $(n=1)$ and GW22 $(n=1)$ hearts, neonate (P6) 4 days post-MI $(n=9)$, and adult (16weeks) 4 weeks post-MI $(n=8)$ mouse hearts. GW, gestation week; $P$, postnatal day; CTO, chronic total occlusion; MI, myocardial infraction; LCA, left coronary artery; LSM, light sheet microscopy. Reported values are mean \pm st dev.

\section{Discussion}

This study is, to our knowledge, the first-time 3D CFD has been used to quantify

562 hemodynamic forces in the adult and neonatal mouse coronary vasculature. The

563 findings here suggest the possible benefit of promoting the growth of fewer, larger

564 arteries and the natural benefit collaterals have in restoring pressure downstream of a 565 stenosis in neonatal hearts.

566 Our whole-organ immunolabeling method identifying the structural relationship of 567 collaterals to coronary artery branches suggested that, in mice, the septal artery could 568 play a more critical role in cardiovascular recovery than previously thought. Recent 569 studies utilizing flattened hearts for whole-mount imaging failed to distinguish the SpA 570 from the $\mathrm{RCA}^{9}$. Here, tissue clearing and Light sheet microscopy allowed visualization

571 of the intact 3D structure and the complete septal artery, revealing its architectural

572 complexity in healthy hearts. Recent studies have outlined SpA development and

573 proposed that the location of its origin from the aorta could significantly impact cardiac

574 recovery during injury models ${ }^{59}$. Our imaging results suggest a potential route to

575 investigate how positional interactions between these branches could impact vascular 576 repair. 
While other studies have performed automatic segmentation and flow modeling 578 of mouse brain and retinal vasculature, there is currently no standard for segmentation and 3D flow simulations in the entire mouse coronary vasculature ${ }^{35}$. We manually

580 segmented over 300 vessels of the adult and over 200 vessels of the neonate coronary

581

582

583

584

585

586

587

588

589

590

591

592

593

594

595

596

597

598

599

600

601

602

603

604

605

606

607 network to ensure accurate representation and high model fidelity for fluid simulations using SimVascular. Manual segmentations are currently required because the signal-tonoise ratios, even with high performing antibodies such as anti-a-SMA, are easily recognized by the human eye, but can cause errors in fully automatic segmentations. Methods are in development to improve automation, such as TubeMap, which utilizes machine learning algorithms to produce high fidelity automated segmentations of the brain vasculature ${ }^{60}$. Future work will focus on using or developing similar methods to automate segmentation for cardiac vasculature.

One major advantage of CFD modeling over ex vivo measurements of experimental samples is the capability to easily modify one feature, i.e. collateral structure, while keeping all other parameters constant. We tested multiple collateral configurations within the same model to understand the relationship of number, position, and diameter on flow recovery, without potential secondary effects from mouse-tomouse variations in coronary structure. In this study, we considered values above $30 \%$ of non-stenotic perfusion levels as being beneficial. This was based on previous in vivo and in vitro studies suggesting that myocardial tissue receiving less than $25-30 \%$ of baseline flow begins to display measurable signs of cardiac dysfunction. For in vivo, when patients were subjected to balloon occlusion of the LCA, only those with greater than approximately $30 \%$ coronary flow index maintained normal ST-segments during electrocardiogram ${ }^{51}$. During Langendorff perfusion preparations, heart rate and left ventricle pressures began recovering to normal values above $25 \%$ of normal perfusion rates $^{52}$. Simulations demonstrated that increasing diameters or positioning collaterals more proximally allowed them to restore a greater volume of cardiac tissue to this $30 \%$ re-perfusion value, more so than increasing numbers of smaller collaterals. One limitation here is that we are not able to test the exact re-perfusion level required for myocyte viability specific to each heart However, we believe that these data are valuable to other scientists in the field studying collateral arteries by giving them general 
608 guidelines of how tested factors affect collateral flow and re-perfusion. With this 609 understanding, physical differences between phenotypes or conditions can be more 610 confidently related to functional differences.

611 It was surprising that even the most effective collateral configuration modeled in 612 the adult-9col, $40 \mu \mathrm{m}$-re-perfused only $\sim 20 \%$ of myocardium above the $30 \%$ ischemic 613 threshold. This is consistent with studies showing significant scar formation in MI 614 models in mice (permanent coronary ligation), even in the presence of collaterals ${ }^{10}$. This 615 underscores the importance of understanding blood flow through experimentally616 induced collateral arteries when considering inducing these vessels as a therapeutic 617 option. Conversely, the virtual collaterals in the neonate with the same characteristics 618 were predicted to have a remarkable ability to shunt flow to the ischemic volume-at619 risk-consistent with studies demonstrating the resilience of neonates after total 620 occlusion $\mathrm{Ml}$ experiments. This, combined with our data that neonates form more 621 numerous and larger collaterals naturally in response to injury, may explain why studies 622 show great recovery post-MI in the neonate via collateral arteries in contrast to adults ${ }^{9}$. 623 This innate difference in collateral function was attributed to a low pressure drop 624 in the neonatal coronary tree compared to adults due to the increased flow in the adult 625 not being compensated by an equal reduction in vascular resistance. It is important to 626 note that at both ages, the pressure at the tips is approximately $40 \mathrm{mmHg}$, indicating 627 that our two models were segmented to a similar extent. The gradual, steady decrease 628 in pressure in the adult arises from the more extensive branching observed compared to 629 the neonate, which is in agreement with studies of pig coronary arteries ${ }^{61}$. While we 630 demonstrated there is very little pressure drop in native neonate coronary arteries, the 631 pressure downstream of the 3D model is expected to abruptly drop to capillary levels. 632 The reduction in total coronary resistance from neonate to adult is in concordance with 633 general trends found with $\mu \mathrm{CT}$ measurements of coronary vessels $>40 \mu \mathrm{m}$ in mice aged 6341 week to 6 weeks old, but quantitatively much greater in our models ${ }^{41,62}$. While these 635 studies described the coronary tree morphology with quantitative scaling laws, they 636 were not able to quantify small diameter arteries, which is evident by the noticeably 637 missing arteries in their 1-week mouse coronary model compared to our Light sheet 
638 imaged P6 hearts. Future work could investigate how these quantitative scaling laws 639 apply to vessels we are able to visualize with our method.

640 We next sought to compare our mouse results to human data. It was unexpected

641 that fetal human hearts contained more native collaterals than the injured neonate and

642 adult mouse as well as diseased adult human hearts, although it is difficult to make

643 comparisons between whole-organ a-SMA staining and angiograms. This finding

644 corresponds with data that suggests collaterals tend to decrease during adolescent

645 years because more were found in the fetal stages compared to neonate humans ${ }^{57,58}$.

646 Since these collaterals are much smaller than those found in adult human diseased

647 hearts via angiogram, it may indicate that small collaterals in the adult heart go

648 undetected. In addition, if we can preserve and enlarge these collaterals in adulthood,

649 they have the potential to greatly improve cardiac perfusion in patients suffering from

650 CAD.

651 Overall, by combining advanced computational and imaging techniques, a novel 652 connection between collateral flow and native morphological differences, and thus 653 pressure distributions, was established. By bridging these two fields, we uncovered how 654 fundamental coronary morphology changes from embryonic to adult in both mouse and 655 humans affect collateral flow. These findings provide insight into why coronary collateral 656 arteries are better suited for recovering from an injury in young hearts compared to old.

\section{Limitations}

One limitation of this study is that we were not able to measure subject-specific aortic flows and pressures for each mouse coronary vasculature in vivo to use for

661 boundary conditions. However, with literature-derived averaged flows scaled to the 662 model size, we expect this to have a very minor effect on the absolute quantities of 663 collateral flow as described here and thus little effect on the overall relative differences 664 between the collateral configurations and ages.

665 Another limitation is that it is not currently possible to measure the outlet 666 pressure of the coronary tree in vivo to validate computational modeling. Due to the 667 small size and inaccessibility of the coronary vasculature in mice, it is challenging to 668 determine in vivo flows and pressures at the pre-capillary level. Pressure 
669 measurements taken from rabbits and dogs suggest that $50-70 \%$ of the pressure is lost 670 when blood reaches the capillary bed ${ }^{63,64}$. In our study, we tuned the outlet boundary 671 conditions such that the resistance of the 3D model was $65 \%$ of the total coronary

672 resistance, which matches expected values from literature ${ }^{63}$. We tested the sensitivity of 673 our results to changes in outlet pressure by adjusting the outlet resistances and found 674 that relative differences between the collateral configurations remained the same.

675 In our modeling approach, we assumed that the downstream arteries and 676 capillaries do not undergo remodeling. While this assumption is not valid for estimating 677 flow in collaterals that form in response to injury, we first sought to develop our 678 modeling to test the effectiveness of pre-existing collaterals. In future studies, we would 679 enhance our computational methods to capture capillary remodeling events that occur 680 due to the re-routing of blood flow.

Methods

683 Animals

$684 \quad$ All mouse colonies were housed and bred in the animal facility at Stanford 685 University in accordance with institutional animal care and use committee (IACUC) 686 guidance.

\section{Immunolabeling and iDISCO clearing}

688 Whole heart vasculature staining was performed following the modified iDISCO+ 689 protocol previously described ${ }^{37,39}$. For all following steps, tissue was always agitated 690 unless noted otherwise. Briefly, animals were perfused with PBS through the dorsal 691 vein, and fixed in $4 \%$ paraformaldehyde (Electron Microscopy Science 15714) at $4^{\circ} \mathrm{C}$ for $6921 \mathrm{hr}$ (neonatal hearts) or 2hr (adult hearts), washed 3X in PBS and stored in PBS with $6930.01 \%$ sodium azide (w/v, Sigma-Aldrich S8032) until ready to process. Hearts were 694 dehydrated in increasing series of methanol/ $\mathrm{ddH}_{2} \mathrm{O}$ dilutions $(20 \%, 40 \%, 60 \%$, $69580 \%, 100 \% 2 \mathrm{X}$ ) for $1 \mathrm{hr}$ each, followed by overnight incubation in $66 \%$ dichloromethane 696 (DCM, Sigma-Aldrich 34856) and 33\% methanol. Next, tissue was washed 2X in 697 methanol $100 \%$ for $4 \mathrm{hrs}$ and bleached overnight at $4^{\circ} \mathrm{C}$ in $5 \%$ hydrogen peroxide 
698 (Sigma-Aldrich 216763) in methanol. Next, the hearts are rehydrated in methanol/ddH$699{ }_{2} \mathrm{O}$ dilutions $(80 \%, 60 \%, 40 \%, 20 \%)$ for $1 \mathrm{hr}$ each, followed by PBS, $0.2 \%$ Triton $\mathrm{X}-100$ 700 PBS (2X) and overnight 20\% dimethyl sulfoxide (DMSO), 2.3\% Glycine (w/v, Sigma 701 G7126), $0.2 \%$ Triton X-100 PBS at $37^{\circ} \mathrm{C}$ for 2 days. For immunostaining, hearts were 702 blocked in 10\% DMSO, 6\% Normal Donkey Serum (NDS, Jackson ImmunoResearch 703 017-000-121) in 0.2\% Triton X-100 for 2 days at 37C. Primary antibodies, aSMA-Cy3 704 conjugated (1:300, Sigma C6198), Connexin-40 (1:300, Alpha diagnostic cx40A), and 705 Podocalyxin (1:1000, R\&D Systems MAB1556) were prepared in PBS with 5\%DMSO, $7063 \%$ NDS in $0.2 \%$ Tween-20, 0.1\% Heparin (w/v, Sigma-Aldrich H3393) and incubated at

$70737^{\circ} \mathrm{C}$ for $4-14$ days. Secondary antibodies conjugated to Alexa 647 (Jackson

708 ImmunoResearch) were matched 1:1 in concentration to their primary target and in 709 prepared in PBS with 3\% NDS in $0.2 \%$ Tween-20, 0.1\% Heparin for the same primary 710 incubation days at $37^{\circ} \mathrm{C}$. Washes after each antibody incubation in PBS with $0.2 \%$

711 Tween-20, 0.1\% Heparin were performed in 30min increment until the end of the day, 712 followed by an overnight wash. Before clearing, samples were embedded in $1 \%$ low713 melting agarose (Sigma-Aldrich A9414) in PBS and dehydrated in methanol/ddd $\mathrm{d}_{2} \mathrm{O}$ 714 dilutions $(20 \%, 40 \%, 60 \%, 80 \%, 100 \% 2 \mathrm{X})$ for $1 \mathrm{hr}$ each and $100 \%$ overnight. Next, 715 hearts were incubated in 66\% DCM and 33\% Methanol for 2.5hrs, followed 2X 30min $716100 \%$ DCM. Finally, samples were cleared in ethyl cinnamate (ECi, Sigma Aldrich 717 112372), manually inverted a few times, and kept at RT in the dark until and after 718 imaging.

719 Light sheet imaging

720 Samples were imaged with LaVision BioTec Ultramicroscope II Light sheet 721 microscope in a quartz cuvette filled with ECi. For imaging, we used a MVX10 zoom 722 body (Olympus) with a 2x objective (pixel size of $3.25 \mu \mathrm{m} / \mathrm{x}, \mathrm{y}$ ) at magnification from

$7230.63 x$ up to $1.6 x$. Up to 1400 images were taken for each heart and the $z$-steps are set

724 to $3.5 \mu \mathrm{m} z$ step size, and light sheet numerical aperture to $0.111 \mathrm{NA}$. Band-pass

725 emission filters (mean $\mathrm{nm} /$ spread) were used, depending on the excited fluorophores:

$726525 / 50$ for autofluorescence; 595/40 for Cy3; 680/30 for AF647 and 835/70 for AF790.

727 Exposure time was $10 \mathrm{~ms}$ for single channel and $25 \mathrm{~ms}$ for multichannel acquisition. 
To determine the approximate volume of myocardium each outlet of the coronary model was responsible for perfusing we used (1) a model of the myocardial tissue as

731 the total volume to be perfused and (2) the outlet coordinates as the seed points for the

732 subvolumes. We used the background signal from the staining to segment the model of 733 the myocardial tissue and the cap centers for the outlet coordinates. Then, we used a

734 Voronoi diagram algorithm to assign subvolumes of the myocardial tissue to each outlet

735 of the coronary model such that every point in the myocardial mesh was assigned to the 736 closest outlet. Distances to the closest outlet were determined using a dijkstra

737 algorithm. By integrating the subvolumes of every outlet on each of the 3 main branches 738 (LCA, RCA, and SpA), we were able to calculate the approximate percentage of the 739 total myocardial volume that each main branch is responsible for. We used these 740 percentages as targets for the flow splits when tuning the outflow boundary conditions 741 for the fluid simulations.

742 We used outlet coordinates instead of centerlines because we were able to 743 better resolve the small coronary arterioles compared to prior studies ${ }^{65}$. This allows us

744 to be certain that myocardial regions close to an outlet are perfused by that outlet, 745 rather than by a large artery nearby that has no outlet nearby.

\section{CFD simulation}

We constructed 3D subject-specific models of the mouse vasculature using 748 SimVascular's cardiovascular modeling pipeline ${ }^{44}$. Briefly, we created path lines for 749 each vessel (about 349 vessels for the adult and 244 for the neonate). Vessels distal to 750 the quaternary branches were ignored. For each path line, the image data was viewed 751 in planes orthogonal to the tangent of the path line to segment the cross-section. Circles 752 were used to approximate the cross-section, as some areas of the vasculature 753 appeared collapsed or deformed. All segmentations were lofted to create a solid model 754 of each branch, and the branches were then unioned together to form a complete 755 geometric model. Finally, the lofted model was discretized into a linear tetrahedral mesh 756 using the commercial meshing library, MeshSim (Simmetrix, Troy, NY), resulting in a 
757 total of 600 thousand and 1.8 million elements for the neonatal and adult models, 758 respectively.

759 After obtaining the mesh, we uniformly scaled it to account for the shrinkage that 760 occurs via iDISCO. We quantified the volume change due to our specific clearing 761 protocol using water displacement pre- and post- iDISCO and found that the heart

762 shrank to about $63 \%$ of its original volume. So, we uniformly scaled the entire volumetric 763 mesh by the inverse (1.58-fold) to ensure that our model faithfully matched the pre764 iDISCO geometry.

765 Inlet boundary conditions were determined as follows. We first determined typical 766 neonate and adult mean pressure and aortic velocity values from literature ${ }^{45,46,66}$ (Table 767 2, rows 1-2). Using the mean aortic velocity and the aortic cross sectional inlet area for 768 each mouse used, a subject-specific aortic inflow was calculated and applied. For 769 pulsatile flow simulations, we constructed representative flow waveforms for an adult 770 mouse by digitizing, smoothing, and scaling a waveform from the literature to match the 771 mean inflow at both ages as calculated previously ${ }^{67}$. At the aortic outlet, we applied a 772 simple RCR boundary condition ${ }^{47}$ (Table 2, rows 3-5). At the coronary artery outlets, we 773 applied a specialized lumped parameter network to represent the downstream coronary 774 vasculature and the time-varying intramyocardial pressure due to the beating cardiac

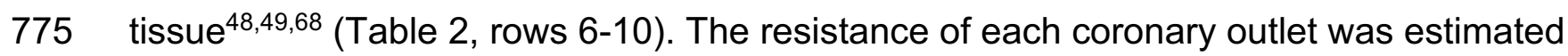
776 using Murray's law and tuned such that each of the 3 main branches (LCA, RCA, and $777 \mathrm{SpA}$ ) had flow splits equal to the percent volume they perfused. To further tune the 778 capacitances and resistances of the coronary boundary conditions to match literature 779 pressure values ${ }^{63,64}$, we used a OD surrogate model for increased efficiency (Fig. S2). 


\begin{tabular}{|c|c|c|c|c|c|}
\hline & Description & $\begin{array}{l}\text { Adult } \\
\text { Value }\end{array}$ & $\begin{array}{l}\text { Adult } \\
\text { Source }\end{array}$ & $\begin{array}{l}\text { Neonate } \\
\text { Value }\end{array}$ & $\begin{array}{c}\text { Neonate } \\
\text { Source }\end{array}$ \\
\hline Qaorta & Aortic flow & $6 * 10^{2}$ & Input & $6.3 * 10^{1}$ & Input \\
\hline$P_{\text {mean }}$ & Mean aortic pressure & $1 * 10^{2}$ & Target & $4 * 10^{1}$ & Target \\
\hline$C_{R C R}$ & $\begin{array}{l}\text { Aortic outlet } \\
\text { capacitance }\end{array}$ & $1 * 10^{-2}$ & Tuned & $1 * 10^{-2}$ & Tuned \\
\hline$R p_{R C R}$ & $\begin{array}{l}\text { Aortic proximal } \\
\text { resistance }\end{array}$ & $4.8 * 10^{-1}$ & Tuned & $5 * 10^{1}$ & Tuned \\
\hline$R d_{R C R}$ & Aortic distal resistance & $1.9 * 10^{1}$ & Tuned & $2.2 * 10^{2}$ & Tuned \\
\hline$R a_{L P N}$ & $\begin{array}{l}\text { Coronary artery } \\
\text { resistance }\end{array}$ & $1.9 * 10^{2}$ & Tuned & $1.7 * 10^{3}$ & Tuned \\
\hline$R u_{L P N}$ & $\begin{array}{l}\text { Coronary microvascular } \\
\text { resistance }\end{array}$ & $3.1 * 10^{2}$ & Tuned & $2.8 * 10^{3}$ & Tuned \\
\hline$R v_{L P N}$ & $\begin{array}{l}\text { Coronary vein } \\
\text { resistance }\end{array}$ & $9.5 * 10^{1}$ & Tuned & $8.5 * 10^{2}$ & Tuned \\
\hline$C a_{L P N}$ & $\begin{array}{l}\text { Coronary arterial } \\
\text { capacitance }\end{array}$ & $\begin{array}{l}3.96 \\
* 10^{-6}\end{array}$ & Tuned & $\begin{array}{l}3.96 \\
* 10^{-6} \\
\end{array}$ & Tuned \\
\hline $\operatorname{Cim}_{L P N}$ & $\begin{array}{l}\text { Intramyocardial } \\
\text { capacitance }\end{array}$ & $\begin{array}{l}3.204 \\
* 10^{-2}\end{array}$ & Tuned & $\begin{array}{l}3.204 \\
* 10^{-2}\end{array}$ & Tuned \\
\hline
\end{tabular}

Table 2. Simulation parameters used for mouse coronary flow modeling. List of parameters used for computational fluid simulations of the adult and neonate coronary arteries. Input and target taken from literature. Tuned parameters were adjusted so that

783 the simulation matched target values given the input. Units: $Q, \frac{\mathrm{mm}^{3}}{\mathrm{~s}} ; P, \mathrm{mmHg} ; C, \frac{\mathrm{s}^{2} \mathrm{~mm}^{4}}{\mathrm{~g}}$;

$784 R, \frac{\mathrm{g}}{\mathrm{mm}^{4} \mathrm{~s}}$.

We globally corrected the viscosity in our pulsatile simulations to $1.25 \mathrm{cP}$ to

787 account for the Fahraeus-Lindqvist effect; this is necessary because the apparent

788 viscosity of blood decreases in very small tube diameters $(<100 \mu \mathrm{m})^{69}$. While this may

789 significantly underestimate the shear stress in the aorta, the pressure drop in the

790 coronaries was more representative and important for the findings presented here (see

791 Limitations).

792 We ran blood flow simulations with rigid walls using the stabilized finite element

793 svSolver code in the open-source SimVascular software package ${ }^{44}$ to determine

794 spatially and temporally resolved hemodynamic values, such as pressure, velocity, and

795 wall shear stress at every node in the computational mesh. Simulations ran for 5 cardiac

796 cycles with timesteps of .0001 seconds, and hemodynamic values were determined 
797 based on the final cardiac cycle. This took approximately 40 hours on 96 cores via

798 XSEDE and 90 hours on 96 cores via Sherlock. Paraview was used for visualization of 799 the results.

800 Virtual collateral placement

801 Virtual collaterals were strategically added to native coronary vasculature to 802 minimize the initial pressure difference of the two points the collateral was connecting.

803 Specifically, based on an initial simulation of the native vasculature (without any virtual

804 collaterals), a pressure distribution was determined. Using this pressure distribution, 805 virtual collaterals were placed such that each connected equal pressure zones. We 806 replicated realistic connections as closely as possible given size and pressure 807 constraints (Fig. S3). The resistance of each collateral configuration was calculated via 808 Poiseuille's Law (equation 1). collaterals in the configuration, and $r$ is the radius of the collateral.

\section{3D Resistance}

813 To calculate the resistance of the 3D model, we first generated vessel centerlines 814 via the Vascular Modeling Tool Kit (VMTK; vmtk.org). Each point in the centerline was 815 identified as a branch segment if a perpendicular cross-section at that point did not 816 intersect with any other centerline point. If the cross-section intersected more than one 817 centerline point, then it was labeled as a junction region. This separated the centerline 818 into junctions and branch segments between junctions. After labeling every point, we 819 determined the parent (upstream) branch segment and child (downstream) branch 820 segments for each junction region. We then calculated the resistance for each branch 821 segment based on the pressure difference from the most proximal to distal point and the 822 flow within that segment from the simulation. Finally, the overall 3D resistance was 823 calculated starting from the most distal branches using a recursive method to add the 824 segment resistances in parallel or in series based on the connectivity. 
826 We utilized the diameter-defined Strahler ordering system to compare

827 morphometric and hemodynamic quantities at similar positions in the coronary tree

828 between the neonate and adult. This system has been used in previous morphometric

829 studies to classify branch segments into orders that describe the hierarchical nature of a

830 vascular tree ${ }^{53,70,71}$. Using the same labels for branch segments and junction regions as

831 in the 3D resistance calculations, we determined the initial Strahler ordering by setting

832 the most distal segments to order 1 and working backwards up the coronary tree to the

833 aorta. Parent segment orders were set to either equal the greater child order if the two

834 children orders were different or incremented by one if the two child orders were the

835 same. Since neither 3D model of the mouse vasculature included all arteries down to

836 the capillary level (only 5 distinct orders here vs. 11 in other studies ${ }^{54}$ ), we translated all

837 the orders by a constant such that the order of the most proximal segment of the

838 coronaries was 12 and the aorta was order 13 to ensure consistency with previous

839 studies. Segments were then re-organized based on their diameter to ensure that

840 unbalanced branching (i.e. a very small vessel branching from a large one) was

841 properly accounted for. To do this, we iteratively moved segments to higher or lower

842 orders such that every segment within an order was within 1 standard deviation of that

843 order's mean diameter. With the final diameter-defined Strahler ordering, we compared

844 quantities such as diameter, length, flow, and pressure between the same orders of the

845 neonate and the adult.

\section{Semi-automated artery tracing}

847 Subsequential images were imported into ImageJ stacks files, these stacks were

848 then converted into 8-bit and resolution reduced to one-fourth the original. Using

849 ImageJ's plug-in, Simple Neurite Tracer, the branch structures of the LCx were able to

850 be drawn by placing seed points along the length of $\alpha-S M A+$ vessels ${ }^{43}$. Once every $\alpha-$

851 SMA+ artery in the LCx branch was completely accounted for within the trace, isolation

852 of the traces was performed by the Fill Out option within the plug-in. The resulting image

853 stack was used as a 3D outline of the arterial structure as the foundation for further 
854 modeling and analysis. After discontinuation of Simple Neurite Tracer, the updated 855 version SNT was used in similar manner as above ${ }^{42}$.

3D Rendering

The non-traced image stack was overlaid with the filled LCx stack using the Add 858 Channel option in Imaris. Pixel dimensions were updated from the non-reduced 16-bit 859 image metadata. The Filament Object Tracer module was used to generate an Imaris 860 customizable 3D LCx branch model. Branch tips and length were measured by 861 automatically generated data under Number of Terminal Points, and Total Length fields, 862 respectively. Branch levels were obtained from the Filaments Branch Hierarchy field. 863 Surface objects in Imaris were used for quantifying the sample heart volumes. 864 Myocardium volume was calculated by creating surface objects surrounding the entire 865 sample surface and objects encompassing the lumen of the ventricles. The volumes of 866 the ventricles were then subtracted from the entire heart volume to result in the 867 myocardium tissue volume.

\section{Murine LCA ligations}

869

Neonatal LCA ligations were performed as previously described ${ }^{9}$ with minimal

871 circulatory arrest and placed in a supine position followed by disinfecting with iodine and

872 ethanol. Dissection was carried through the pectoralis major and minor muscles, and

873 the thoracic cavity was entered via the 4th intercostal space. The LCA was identified

874 and ligated at with a doble knot using 8-0 nylon suture, leaving the LCx intact. The chest 875 muscle and skin were then closed (independently) with interrupted 7-0 prolene sutures.

876 The neonate was then allowed to recover at $37^{\circ} \mathrm{C}$ warm plate and, when conscious, 877 returned to its mother's care.

$878 \quad$ Adult mice were performed as previously described ${ }^{72}$. Adult mice were subjected 879 to permanent coronary artery ligation, under anesthesia using initially $1.5 \%-4 \%$ 880 isoflurane chamber for induction. The chest cavity was opened, and a 7-0 silk suture 881 was placed around the left coronary artery, with occlusion verified by blanching of the 
882 underlying myocardium. The chest was then sutured closed. Following surgery, 883 Buprenorphine $(0.1 \mathrm{mg} / \mathrm{kg})$ was used as an analgesic.

\section{Immunohistochemistry and confocal microscopy}

885 Neonatal or adult hearts were fixed in $4 \%$ PFA overnight at $4{ }^{\circ} \mathrm{C}$, and then 886 cryopreserved in $30 \%$ sucrose in PBS for 1 day at $4^{\circ} \mathrm{C}$. The following day, coronal heart 887 sections ( $50 \mu \mathrm{m}$ in thickness) were cut on a cryostat. Sections were rinsed $3 \mathrm{X}$ with PBS, 888 blocked in $5 \%$ NDS, $0.5 \%$ Triton X-100 in PBS for $1 \mathrm{hr}$ at RT, and then incubated with 889 aSMA-Cy3 conjugated (1:300, Sigma C6198) in 0.5\% Triton X-100 in PBS overnight at $89044^{\circ} \mathrm{C}$. Next, sections were rinsed $3 \mathrm{X}$ in $0.5 \%$ Triton X-100 in PBS and then mounted on

891 slides and covered with Fluoromount G (SouthernBiotech 0100-01). Tissue was imaged 892 using inverted Zeiss LSM-700 confocal microscope at 5x objective. Digital images were 893 captured with Zeiss Zen software and measured using ImageJ.

\section{Human hearts}

Under IRB approved protocols, human fetal hearts were collected for 896 developmental analysis from elective terminations ${ }^{73}$. Gestational age was determined 897 by standard dating criteria by last menstrual period and ultrasound ${ }^{74}$. Tissue was 898 processed within $1 \mathrm{hr}$ following procedure. Tissue was extensively rinsed with cold, 899 sterile PBS while placed on ice, followed by incubation in sterile $4 \%$ PFA for $4 \mathrm{hrs}$ at $4{ }^{\circ} \mathrm{C}$ 900 before further iDISCO processing. Pregnancies complicated by multiple gestations and 901 known fetal or chromosomal anomalies were excluded.

$902 \quad H u m a n$ adult samples were acquired from the Stanford Catheterization 903 Angiography Laboratory. All patients displayed symptoms of chronic angina and were 904 scheduled to receive conventional coronary angiography, which was performed 905 according to local clinical standards. Collateral number and size were confirmed by an 906 experienced cardiologist.

\section{Statistical Analysis}

908 Graphs represent mean values obtained from multiple experiments and error 909 bars represent standard deviation. Unpaired Student's $t$ test was used to compare 
910 groups within an experiment and the level of significance were assigned to statistics in 911 accordance with their $\mathrm{p}$ values ( 0.05 flagged as $*, 0.01$ flagged as $* *$, less than 0.001

912 flagged as $* * *$, less than 0.0001 flagged as $* * * *)$. All graphs were generated using 913 GraphPad Prism software. Error bars represent \pm standard deviation. 


\section{References}

915 1. Go, A. S. et al. Heart Disease and Stroke Statistics - 2014 Update: A report from 916 the American Heart Association. Circulation vol. 129 (2014).

917 2. Zimarino, M., D'andreamatteo, M., Waksman, R., Epstein, S. E. \& De Caterina, R. 918 The dynamics of the coronary collateral circulation. Nature Reviews Cardiology 919 vol. 11 191-197 (2014).

920 3. Habib, G. B. et al. Influence of coronary collateral vessels on myocardial infarct 921 size in humans. Results of Phase I thrombolysis in myocardial infarction (TIMI) $922 \quad$ trial. Circulation 83, 739-746 (1991).

923 4. Helfant, R. H., Vokonas, P. S. \& Gorlin, R. Functional Importance of the Human 924 Coronary Collateral Circulation. N. Engl. J. Med. 284, 1277-1281 (1971).

925 5. Kim, E. K. et al. A protective role of early collateral blood flow in patients with ST926 segment elevation myocardial infarction. Am. Heart J. 171, 56-63 (2016).

927 6. Meier, P. et al. The impact of the coronary collateral circulation on outcomes in 928 patients with acute coronary syndromes: Results from the ACUITY trial. Heart $929 \quad 100,647-651$ (2014).

930 7. Red-Horse, K. \& Das, S. New Research Is Shining Light on How Collateral 931 Arteries Form in the Heart: a Future Therapeutic Direction? Current Cardiology Reports vol. 23 (2021).

8. Maxwell, M. P., Hearse, D. J. \& Yellon, D. M. Species variation in the coronary

942 11. He, L. et al. Genetic lineage tracing discloses arteriogenesis as the main collateral circulation during regional myocardial ischaemia: A critical determinant of the rate of evolution and extent of myocardial infarction. Cardiovascular Research vol. 21 737-746 (1987).

9. Das, S. et al. A Unique Collateral Artery Development Program Promotes mechanism for collateral growth in the mouse heart. Cardiovasc. Res. 109, 419430 (2016). 
945 12. Porrello, E. R. et al. Regulation of neonatal and adult mammalian heart regeneration by the miR-15 family. Proc. Natl. Acad. Sci. U. S. A. 110, 187-192 (2013).

13. Hara, M. et al. Impact of coronary collaterals on in-hospital and 5-year mortality after ST-elevation myocardial infarction in the contemporary percutaneous coronary intervention era: a prospective observational study. BMJ Open 6 , e011105 (2016).

14. Lucitti, J. L. et al. Variants of Rab GTPase-effector binding protein-2 cause variation in the collateral circulation and severity of stroke. Stroke 47, 3022 (2016).

15. Billinger, M. et al. Physiologically assessed coronary collateral flow and adverse cardiac ischemic events: A follow-up study in 403 patients with coronary artery disease. J. Am. Coll. Cardiol. 40, 1545-1550 (2002).

16. Pohl, T. et al. Frequency distribution of collateral flow and factors influencing collateral channel development: Functional collateral channel measurement in 450 patients with coronary artery disease. J. Am. Coll. Cardiol. 38, 1872-1878 (2001).

17. Traupe, T., Gloekler, S., De Marchi, S. F., Werner, G. S. \& Seiler, C. Assessment of the human coronary collateral circulation. Circulation vol. 122 1210-1220 (2010).

18. Factor, S. M. et al. Coronary microvascular abnormalities in the hypertensivediabetic rat. A primary cause of cardiomyopathy? Am. J. Pathol. 116, 9 (1984).

19. Braun, E. J. Intrarenal blood flow distribution in the desert quail following salt loading. Am. J. Physiol. 231, 1111-1118 (1976).

21. Vasquez, S. X. et al. Optimization of MicroCT Imaging and Blood Vessel Diameter Quantitation of Preclinical Specimen Vasculature with Radiopaque Polymer Injection Medium. PLoS One 6, e19099 (2011).

974 22. Merz, S. F. et al. Contemporaneous 3D characterization of acute and chronic myocardial I/R injury and response. Nat. Commun. 10, 1-14 (2019). 
976 23. Honeycutt, S. E. \& O'Brien, L. L. Injection of Evans blue dye to fluorescently label and image intact vasculature. Biotechniques 70, (2020).

978 24. Les, A. S. et al. Quantification of hemodynamics in abdominal aortic aneurysms during rest and exercise using magnetic resonance imaging and computational fluid dynamics. Ann. Biomed. Eng. 38, 1288-1313 (2010).

25. Seo, J., Ramachandra, A.

A. B., Boyd, J., Marsden, A. L. \& Kahn, A. M.

982

983

984 Computational Evaluation of Venous Graft Geometries in Coronary Artery Bypass Surgery. Semin. Thorac. Cardiovasc. Surg. (2021)

985 doi:10.1053/j.semtcvs.2021.03.007.

26. Min, J. K. et al. Diagnostic Accuracy of Fractional Flow Reserve From Anatomic CT Angiography. JAMA 308, 1237-1245 (2012).

27. Zhao, S. et al. Patient-specific computational simulation of coronary artery bifurcation stenting. Sci. Reports $202111111,1-17$ (2021).

28. Shad, R. et al. Patient-Specific Computational Fluid Dynamics Reveal Localized Flow Patterns Predictive of Post-Left Ventricular Assist Device Aortic Incompetence. Circ. Hear. Fail. 737-745 (2021) doi:10.1161/CIRCHEARTFAILURE.120.008034.

29. Su, B. et al. Numerical investigation of blood flow in three-dimensional porcine left 995

30. Peiffer, V., Rowland, E. M., Cremers, S. G., Weinberg, P. D. \& Sherwin, S. J. Effect of aortic taper on patterns of blood flow and wall shear stress in rabbits: Association with age. Atherosclerosis 223, 114-121 (2012).

31. Lindsey, S. E. et al. Growth and hemodynamics after early embryonic aortic arch occlusion. Biomech. Model. Mechanobiol. 14, 735-751 (2015).

32. Vedula, $V$. et al. A method to quantify mechanobiologic forces during zebrafish cardiac development using 4-D light sheet imaging and computational modeling. PLOS Comput. Biol. 13, e1005828 (2017).

33. Suo, J. et al. Hemodynamic Shear Stresses in Mouse Aortas Implications for Atherogenesis Materials and Methods Geometry Data Acquisition of the Mouse 1006 Aorta. (2007) doi:10.1161/01.ATV.0000253492.45717.46. 
1007 34. Shannon, A. T. \& Mirbod, P. Three-dimensional flow patterns in the feto-placental vasculature system of the mouse placenta. Microvasc. Res. 111, 88-95 (2017).

35. Bernabeu, M. O. et al. Computer simulations reveal complex distribution of haemodynamic forces in a mouse retina model of angiogenesis. J. R. Soc. Interface 11, (2014).

36. Acuna, A. et al. Computational Fluid Dynamics of Vascular Disease in Animal Models. J. Biomech. Eng. 140, 0808011 (2018).

37. Rios Coronado, P. E. \& Red-Horse, K. Enhancing cardiovascular research with whole-organ imaging. Curr. Opin. Hematol. 28, 214-220 (2021).

38. Renier, N. et al. IDISCO: A simple, rapid method to immunolabel large tissue samples for volume imaging. Cell 159, 896-910 (2014).

39. Renier, N. et al. Mapping of Brain Activity by Automated Volume Analysis of Immediate Early Genes. Cell 165, 1789-1802 (2016).

40. Pan, C. et al. Shrinkage-mediated imaging of entire organs and organisms using uDISCO. Nat. Methods 13, 859-867 (2016).

41. Feng, Y. et al. Bifurcation asymmetry of small coronary arteries in juvenile and adult mice. Front. Physiol. 9, 519 (2018).

42. Arshadi, C., Günther, U., Eddison, M., Harrington, K. I. S. \& Ferreira, T. A. SNT: a unifying toolbox for quantification of neuronal anatomy. Nat. Methods 18, 374-377 (2021).

43. Longair, M. H., Baker, D. A. \& Armstrong, J. D. Simple Neurite Tracer: open source software for reconstruction, visualization and analysis of neuronal processes. Bioinformatics 27, 2453-2454 (2011).

44. Updegrove, A. et al. SimVascular: An Open Source Pipeline for Cardiovascular Simulation. Annals of Biomedical Engineering vol. 45 525-541 (2017).

46. Huo, Y., Guo, X. \& Kassab, G. S. The flow field along the entire length of mouse aorta and primary branches. Ann. Biomed. Eng. 36, 685-699 (2008).

1037 47. Vignon-Clementel, I. E., Figueroa, C. A., Jansen, K. E. \& Taylor, C. A. Outflow 
1038

1039

1040

1041

1042

1043

1044

1045

1046

1047

1048

1049

1050

1051

1052

1053

1054

1055

1056

1057

1058

1059

1060

1061

1062

1063

1064

1065

1066

1067

1068

boundary conditions for 3D simulations of non-periodic blood flow and pressure fields in deformable arteries. Comput. Methods Biomech. Biomed. Engin. 13, 625-640 (2010).

48. Kim, H. J. et al. Patient-Specific Modeling of Blood Flow and Pressure in Human Coronary Arteries. doi:10.1007/s10439-010-0083-6.

49. Tran, J. S., Schiavazzi, D. E., Ramachandra, A. B., Kahn, A. M. \& Marsden, A. L. Automated tuning for parameter identification and uncertainty quantification in multi-scale coronary simulations. Comput. Fluids 142, 128-138 (2017).

50. Huang, Y., Guo, X. \& Kassab, G. S. Axial nonuniformity of geometric and mechanical properties of mouse aorta is increased during postnatal growth. Am. J. Physiol. - Hear. Circ. Physiol. 290, 657-664 (2006).

51. Seiler, C., Fleisch, M., Garachemani, A. \& Meier, B. Coronary collateral quantitation in patients with coronary artery disease using intravascular flow velocity or pressure measurements. J. Am. Coll. Cardiol. 32, 1272-1279 (1998).

52. Stoner, J. D., Angelos, M. G. \& Clanton, T. L. Myocardial contractile function during postischemic low-flow reperfusion: critical thresholds of $\mathrm{NADH}$ and $\mathrm{O}_{2}$ delivery. Am. J. Physiol. Circ. Physiol. 286, H375-H380 (2004).

53. Huang, W., Yen, R. T., McLaurine, M. \& Bledsoe, G. Morphometry of the human pulmonary vasculature. $h$ ttps://doi.org/10.1152/jappl.1996.81.5.2123 81, 21232133 (1996).

54. Kassab, G. S., Rider, C. A., Tang, N. J. \& Fung, Y. C. B. Morphometry of pig coronary arterial trees. Am. J. Physiol. - Hear. Circ. Physiol. 265, (1993).

55. Wustmann, K., Zbinden, S., Windecker, S., Meier, B. \& Seiler, C. Is there functional collateral flow during vascular occlusion in angiographically normal coronary arteries? Circulation 107, 2213-2220 (2003).

56. Meier, P. et al. The collateral circulation of the heart. BMC Med. $201311111,1-7$ (2013).

57. Reiner, L., Molnar, J., Jimenez, F. A. \& Freudenthal, R. R. Interarterial coronary anastomoses in neonates. Arch. Pathol. 71, 103-112 (1961).

58. Bloor, C. M., Keefe, J. F. \& Browne, M. J. Intercoronary anastomoses in congenital heart disease. Circulation 33, 227-231 (1966). 
1069

1070

1071

1072

1073

1074

1075

1076

1077

1078

1079

1080

1081

1082

1083

1084

1085

1086

1087

1088

1089

1090

1091

1092

1093

1094

1095

1096

1097

1098

1099

59. Kolesová, H., Bartoš, M., Hsieh, W. C., Olejníčková, V. \& Sedmera, D. Novel approaches to study coronary vasculature development in mice. Dev. Dyn. 247, 1018-1027 (2018).

60. Kirst, C. et al. Mapping the Fine-Scale Organization and Plasticity of the Brain Vasculature. Cell 180, 780-795.e25 (2020).

61. Mittal, N. et al. Analysis of blood flow in the entire coronary arterial tree. Am. J. Physiol. - Hear. Circ. Physiol. 289, 439-446 (2005).

62. Huo, Y. et al. Growth, ageing and scaling laws of coronary arterial trees. J. R. Soc. Interface 12, (2015).

63. Chilian, W. M., Eastham, C. L. \& Marcus, M. L. Microvascular distribution of coronary vascular resistance in beating left ventricle. Am. J. Physiol. - Hear. Circ. Physiol. 251, (1986).

64. Nellis, S. H., Liedtke, A. J. \& Whitesell, L. Small coronary vessel pressure and diameter in an intact beating rabbit heart using fixed-position and free-motion techniques. Circ. Res. 49, 342-353 (1981).

65. Malkasian, S., Hubbard, L., Dertli, B., Kwon, J. \& Molloi, S. Quantification of vessel-specific coronary perfusion territories using minimum-cost path assignment and computed tomography angiography: Validation in a swine model. $J$.

Cardiovasc. Comput. Tomogr. 12, 425-435 (2018).

66. Van Doormaal, M. A. et al. Haemodynamics in the mouse aortic arch computed from MRI-derived velocities at the aortic root. J. R. Soc. Interface 9, 2834-2844 (2012).

67. Hartley, C. J., Reddy, A. K., Michael, L. H., Entman, M. L. \& Taffet, G. E. Coronary flow reserve as an index of cardiac function in mice with cardiovascular abnormalities. in 2009 Annual International Conference of the IEEE Engineering in Medicine and Biology Society 1094-1097 (IEEE, 2009).

doi:10.1109/IEMBS.2009.5332488.

68. Sankaran, S. et al. Patient-specific multiscale modeling of blood flow for coronary artery bypass graft surgery. Ann. Biomed. Eng. 40, 2228-2242 (2012).

69. Fåhræus, R. \& Lindqvist, T. THE VISCOSITY OF THE BLOOD IN NARROW CAPILLARY TUBES. Am. J. Physiol. Content 96, 562-568 (1931). 
1100 70. Kassab, G. S., Rider, C. A., Tang, N. J. \& Fung, Y. C. B. Morphometry of pig coronary arterial trees. Am. J. Physiol. - Hear. Circ. Physiol. 265, (1993).

1102 71. Dong, M. et al. Image-based scaling laws for somatic growth and pulmonary artery morphometry from infancy to adulthood. Am. J. Physiol. - Hear. Circ. Physiol. 319, H432-H442 (2020).

72. Raftrey, B. et al. Dach1 Extends Artery Networks and Protects Against Cardiac Injury. Circ. Res. (2021) doi:10.1161/circresaha.120.318271.

73. Cunningham, F. G. et al. Abortion. in Williams Obstetrics, 25e (McGraw-Hill Education, 2018).

74. Cunningham, F. G. et al. Prenatal Care. in Williams Obstetrics, 25e (McGraw-Hill Education, 2018).

Acknowledgements

We thank Andrew Olson and Marco Howard for technical support of Light sheet imaging, and Hanjay Wang for advice on surgical procedures. S.A is supported by BioX Bowes Fellowship. P.E.R.C is supported by the NIGMS of the National Institutes of Health (NIH T32GM007276) and NSF-GRFP (DGE-1656518). M.L.D is supported by the NSF-GRFP (DGE-1656518). D. B. is supported by the Department of Defense CMDRP in Congenital Heart Disease (W81XWH-16-1-0727). K.N. is supported by the $\mathrm{NIH} / \mathrm{NHLBL}$ (R01HL141712; R01HL146754). A.L.M is supported by NIH (R01EB018302) and NSF Award (1663671). K.R.-H. is supported by the NIH/NHLBL (R01-HL128503) and the New York Stem Cell Foundation (NYSCF-Robertson 1121 Investigator).

\section{Contributions}

S.A., P.E.R.C., A.L.M., and K.R.-H. conceived and designed the project. S.A., P.E.R.C., A.N.L.S.-Q., and C.K.C. performed experiments. S.A, P.E.R.C., A.N.L.S.-Q., A.S., and A.M.H. analyzed data. S.A. performed fluid simulations. P.E.R.C., B.C.R., M.Z., and D.B. performed murine cardiac injury studies. K.N. and A.M.P. contributed human adult and fetal samples, respectively. S.A., M.L.D., and M.P. provided analysis tools. S.A. and P.E.R.C. prepared figures. S.A., P.E.R.C., A.L.M., and K.R.-H. wrote the 1129 manuscript. 


\section{Supplementary Files}

This is a list of supplementary files associated with this preprint. Click to download.

- Supplemental.pdf 